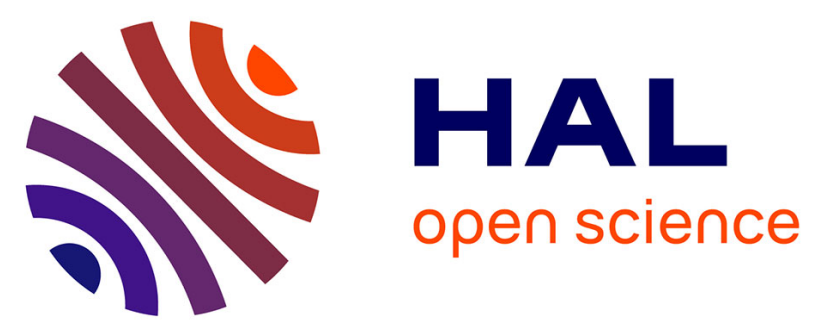

\title{
Multi-scale optimisation of thin-walled structures by considering a global/local modelling approach
}

Michele I. Izzi, Marco Montemurro, Anita Catapano, Daniele Fanteria, Jérôme Pailhes

\section{- To cite this version:}

Michele I. Izzi, Marco Montemurro, Anita Catapano, Daniele Fanteria, Jérôme Pailhes. Multi-scale optimisation of thin-walled structures by considering a global/local modelling approach. Proceedings of the Institution of Mechanical Engineers, Part G: Journal of Aerospace Engineering, 2020, 10.1177/0954410020939338 . hal-02910815

\section{HAL Id: hal-02910815 https://hal.inrae.fr/hal-02910815}

Submitted on 22 Sep 2020

HAL is a multi-disciplinary open access archive for the deposit and dissemination of scientific research documents, whether they are published or not. The documents may come from teaching and research institutions in France or abroad, or from public or private research centers.
L'archive ouverte pluridisciplinaire HAL, est destinée au dépôt et à la diffusion de documents scientifiques de niveau recherche, publiés ou non, émanant des établissements d'enseignement et de recherche français ou étrangers, des laboratoires publics ou privés. 


\title{
Multi-scale optimisation of thin-walled structures by considering a global/local modelling approach ${ }^{\star}$
}

\author{
Michele Iacopo Izzi ${ }^{\mathrm{a}}$, Marco Montemurro ${ }^{\mathrm{a}, *}$, Anita Catapano $^{\mathrm{b}}$, Daniele Fanteria ${ }^{\mathrm{c}}$, Jérôme Pailhès ${ }^{\mathrm{a}}$ \\ ${ }^{a}$ Arts et Métiers Institute of Technology, Université de Bordeaux, CNRS, INRA, Bordeaux INP, HESAM Université, \\ I2M UMR 5295, F-33405 Talence, France \\ ${ }^{b}$ Bordeaux INP, Université de Bordeaux, Arts et Métiers Institute of Technology, CNRS, INRA, HESAM Université, \\ I2M UMR 5295, F-33405 Talence, France \\ ${ }^{c}$ University of Pisa, Department of Civil and Industrial Engineering - Aerospace division, via G. Caruso 8, 56122 \\ Pisa, Italy
}

\begin{abstract}
In this work, a design strategy for optimising thin-walled structures based on a global-local finite element (FE) modelling approach is presented. The preliminary design of thin-walled structures can be stated in the form of a constrained non-linear programming problem (CNLPP) involving requirements of different nature intervening at the different scales of the structure. The proposed multi-scale optimisation (MSO) strategy is characterised by two main features. Firstly, the CNLPP is formulated in the most general sense by including all design variables involved at each pertinent scale of the problem. Secondly, two scales (with the related design requirements) are considered: i) the structure macroscopic scale, where low-fidelity FE models are used; ii) the structure mesoscopic scale (or component-level), where more accurate FE models are involved. In particular, the mechanical responses of the structure are evaluated at both global and local scales, avoiding the use of approximated analytical methods. The MSO is here applied to the least-weight design of an aluminium fuselage barrel of a wide-body aircraft. Fully parametric global and local FE models are interfaced with an in-house metaheuristic algorithm. Refined local FE models are created only for critical regions of the structure, automatically detected during the global analysis, and linked to the global one thanks to the implementation of a sub-modelling approach. The whole process is completely automated and, once set, it does not need any further user intervention.
\end{abstract}

Keywords: Optimisation, Genetic algorithms, Fuselage, Stiffened panels, Finite Element Method, Global/local modelling approach

This is a pre-print of an article published in Proceedings of the Institution of Mechanical Engineers, Part G.

The final authenticated version is available online at:

https://doi.org/10.1177/0954410020939338

*This paper presents part of the activities carried out within the research project PARSIFAL (Prandtlplane ARchitecture for the Sustainable Improvement of Future AirpLanes), which has been funded by the European Union under the Horizon 2020 Research and Innovation Program (Grant Agreement n.723149)

${ }^{*}$ Corresponding author. Tel.: +33 556845 422, Fax.: +33 540006964.

Email address: marco.montemurro@ensam.eu, marco.montemurro@u-bordeaux.fr (Marco Montemurro) 


\section{Introduction}

Mass control is a major concern in the design of airplanes. For this reason, the design of aircraft structures is often formulated as a constrained non-linear programming problem (CNLPP). The main objective is the mass minimisation subject to a given number of design requirements stated as optimisation constraints. "Semi-monocoque" structures have soon became a standard choice for both the fuselage and the wing thanks to their favourable stiffness-to-weight ratio. Due to their nature, the design criteria for such structures involve both local phenomena (i.e. at the scale of the single component such as a stiffened panel) and global ones (i.e. at the scale of the whole structure). For this reason, a multi-scale modelling approach reveals necessary to properly describe the interdependency of the different phenomena and, consequently, a suitable multi-scale optimisation (MSO) strategy, integrating such a modelling approach, shall be defined.

The preliminary phase of aircraft structural design is mostly based on analytical or semiempirical methods. Such methods have been developed since 40s especially in USA [1-3] and have been continuously improved during the years until becoming an established reference for aircraft designers [4-7]. These methods are based on several simplifying hypotheses. For example, when dealing with the fuselage design, at the global scale circular cross-sections are considered, whose geometry and boundary conditions (BCs) are symmetric with respect to the aircraft longitudinal plane. Stringers are taken into account by considering an homogeneous skin of equivalent thickness (greater than the true thickness of the skin) or by considering lumped models in which a group of stringers is merged in one rod element. When pressurisation is taken into account, the classical equations for axial-symmetrical infinite vessels with regularly spaced frames are used [7]. At the stiffened panel scale, for the calculation of buckling loads, plane or curved plates with uniformly loaded edges and perfect BCs (usually in the form of simply supported edges) are considered; the stringers are considered as isolated elements and the Euler column buckling equations are used $[6,8]$.

Preliminary design procedures for thin-walled structures have been developed integrating the aforementioned methods into sequential [8] or iterative procedures [9-11]. The solution is searched by means of gradient-based algorithms using an initial guess solution set by means of handbook methods. In order to properly exploit the effectiveness of the gradient-based algorithm, the number of design variables is reduced by "slaving" or "linking" them together by enforcing fabrication requirements or using experience or simplified empirical rules. Of course, this approach extremely shrinks the design domain.

Several works on improved analytical or numerical methods for predicting the mechanical response of stiffened panels, especially regarding the buckling and post-buckling behaviour of such components [12-14] can be found in literature. However, the aforementioned simplifying hypotheses are still used.

In 1972, Sobieszczanski and Loendorf [15] proposed a mixed optimisation method in which a "lumped" global FE model (GFEM) was used instead of analytical formulae to evaluate the stiffness of the fuselage in order to obtain a better approximation of the loads to be used on local buckling evaluations performed using classical analytical methods. Similarly, Fischer et al. [16] proposed a multi-level framework for optimisation of lightweight structures in which a simplified GFEM evaluates the average membrane and bending loads for the local optimisation of panels performed using VICONOPT, a program based on analytical solutions for prismatic plane panels with simple supported edges or periodic BCs. An analogous work is presented by Grihon et al. [9] in which the tool ASSIST for the buckling and post-buckling analyses based on engineering formulae was employed. They also considered the possibility of using a surrogate model to speed-up the process. 
A surrogate model is also adopted in [17] for the multi-scale optimisation of a fuselage barrel. In this case the surrogate model is used for buckling failure evaluations and it is generated by means of buckling analyses on a parametric high fidelity local FE model (LFEM) of an isolated stiffened panel with idealised BCs. They showed the poor accuracy of the local buckling analyses by highlighting, thus, the detrimental effect of the idealised BCs.

In all the aforementioned works the local analysis (and the related optimisation) is performed by considering fixed internal loads resulting from the GFEM, hence neglecting stress redistribution due to the change of geometry at the upper scale, i.e. that of the whole structure. Therefore, the main limitation of such design procedures is related to a poor evaluation of the mechanical response of the structure, due either to the use of simplified models or to the approximation of the BCs in the passage from the GFEM to the LFEM. In order to overcome these limitations, the utilisation of a proper global/local (GL) modelling approach in the framework of the MSO of thin-walled structures is proposed in this work.

GL modelling approaches allow the assessment of phenomena involved at the-component level through the use of LFEMs with realistic BCs derived from the GFEM. Both models have affordable computational costs so that they can be integrated into an optimisation strategy [18]. GL modelling approaches have been investigated mainly during 80s and 90s [19-25]. During the last two decades, as a consequence of the improved computational capabilities, GL approaches have been developed and used also to assess more complex phenomena like local plasticity [26], crack propagation [27], delamination in composite structures [28] and strong non-linear phenomena as well. Most of these works are developed for specific applications and their implementation needs the creation of specific tools not available in commercial FE software. Apart the GL modelling strategies that do not make use of commercial FE software, the rest of GL approaches can be divided into two categories: sub-modelling [22-25] and condensation/zooming techniques [19, 20].

In the usual work-flow of sub-modelling GL approaches, firstly a low fidelity linear analysis on a GFEM with a coarse mesh is run to identify one or more zones of interest (ZOIs). Then a refined LFEM is created for each ZOI. The refined LFEM can also take into account complex nonlinear phenomena. Then, a second analysis is performed only on the LFEM imposing displacements provided by the GFEM as BCs. Moreover, iterative stages can be added if the stress redistribution due to local effects is considered non-negligible. An alternative formulation making use of forces and stiffness instead of displacements in the information transfer between GFEM and LFEM has been proposed too $[21]$.

The condensation/zooming technique $[19,20]$ differs from the sub-modelling approach at the second step where the refined LFEM is solved together with a condensed version of the "out-of-interest" region of the GFEM, introduced in the form of a super-element. From a computational point of view the procedure is more expensive than the sub-modelling approach but it does not require an iterative process.

In this work, a MSO of thin-walled aircraft structures making use of the sub-modelling GL approach is presented. The MSO strategy is characterised by two main features: on the one hand, the full set of design variables, at each relevant scale of the problem, is considered in the design process without additional simplifying hypothesis, widening in this way the design space and, consequently, the possibility to find a true global optimum solution. On the other hand, all the design criteria and requirements involved into the problem formulation are evaluated by means of both GFEM and LFEMs through a suitable GL modelling approach. Computational time is kept low by verifying local responses only on the most critical ZOIs. To this purpose, pertinent design criteria are introduced into the GFEM to automatically identify the ZOIs and build the related refined LFEMs. 
The solution search is carried out by means of the special genetic algorithm (GA) ERASMUS (EvolutionaRy Algorithm for optimiSation of ModUlar Systems) [29] which can deal with CNLPPs defined over a design space of variable dimension.

The effectiveness of the proposed MSO strategy is proven on a meaningful real-world engineering problem: the least-weight design of a fuselage barrel belonging to the aft part of a wide-body aircraft that undergoes multiple loading conditions and subject to constraints of different nature.

The paper is organised as follows. A general description of the design problem, the underlying hypotheses and the driving design criteria is given in Sec. 2. The mathematical formulation of the multi-scale design problem and the adopted numerical strategy are discussed in Sec. 3. The details on the FE models and the implementation of the GL approach are presented in Sec. 4. Numerical results are shown in Sec. 5. Finally Sec. 6 ends the paper with some conclusions and perspectives.

\section{Multi-scale least-weight design of a metallic fuselage section: problem description}

The MSO strategy presented in this study is applied to the least-weight design of an aluminium fuselage barrel of a wide-body aircraft. The fuselage barrel has a circular cross-section and is located between the wing rear spar and the tail, as shown in Fig. 1. The fuselage barrel is clamped at the rear spar section (section A) and loads coming from the tail are applied to section B. Payload weight and pressurisation are also taken into account. More details on the BCs and the load cases considered in the design process will be given in Secs. 2.1 and 2.2.

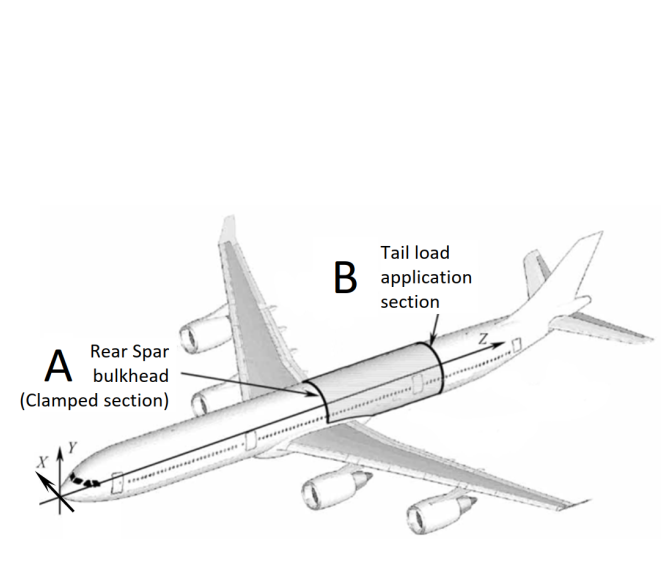

(a)

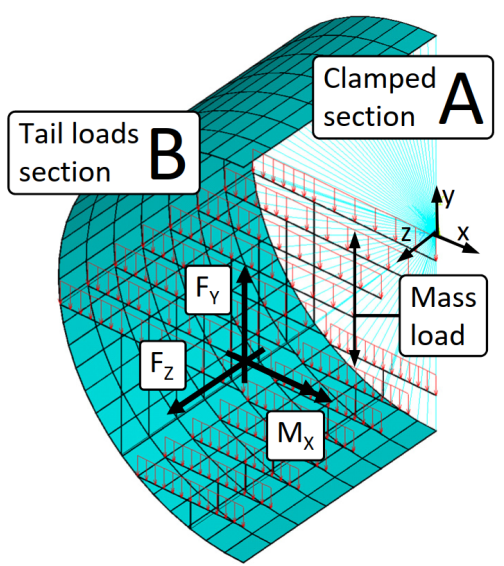

(b)

Figure 1: Location of the fuselage barrel [30] (a) and detail of the applied BCs (b).

The main geometrical parameters of the fuselage cross-section, the structural architecture and loads are taken from $[11,30]$ where an iterative design procedure integrating several analytical methods was presented. Their solution is hereafter referred as literature solution (LSol).

The main geometrical parameters of the fuselage barrel are reported in Tab. 1. The remaining data necessary to define LSol can be found in Tab. 2: the meaning of some of these parameters is explained in Sec. 3.1. The generic stiffened panel geometry considered in this study is shown in Fig. 2. It is composed of hat-shaped stringers and floating frames with a Z-shaped cross-section attached to the skin by means of "shear tie" components; no "stringer tie" or "tear strap" components are present. Floor beams with an I-shaped cross-section and tubular struts complete the set of structural components. 


\begin{tabular}{|c|c|}
\hline Component & Value \\
\hline Fuselage diameter $[\mathrm{mm}]$ & 5640 \\
\hline Number of bays & 7 \\
\hline Bay pitch $[\mathrm{mm}]$ & 500 \\
\hline Upper-deck floor vertical position $[\mathrm{mm}]^{\dagger}$ & -152 \\
\hline Lower-deck floor vertical position $[\mathrm{mm}]^{\dagger}$ & -2130 \\
\hline Struts position on upper-deck floor beam * & $1 / 3$ \\
\hline Struts position on lower-deck floor beam * & $1 / 4$ \\
\hline
\end{tabular}

Table 1: Main geometrical parameters of the fuselage barrel.

\begin{tabular}{|c|c|c|c|}
\hline Component & & Value & \\
\hline Frame flange width $\left(w_{3}^{\mathrm{Fr}}\right)[\mathrm{mm}]$ & & 35.0 & \\
\hline Frame web height $\left(w_{3}^{\mathrm{Fr}}\right)[\mathrm{mm}]$ & & 165.0 & \\
\hline Frame thickness $\left(t^{\mathrm{Fr}}\right)[\mathrm{mm}]$ & & 1.5 & \\
\hline Cabin floor beams web height $[\mathrm{mm}]$ & & 240.0 & \\
\hline Cabin floor beams flange width $[\mathrm{mm}]$ & & 156.0 & \\
\hline Cabin floor beams thickness [mm] & & 2.5 & \\
\hline Cargo floor beams web height $[\mathrm{mm}]$ & & 180.0 & \\
\hline Cargo floor beams flange width [mm] & & 60.0 & \\
\hline Cargo floor beams thickness [mm] & & 1.5 & \\
\hline Struts external diameter $[\mathrm{mm}]$ & & 21.5 & \\
\hline Struts internal diameter [mm] & & 15.5 & \\
\hline Component & Top & Lateral & Bottom \\
\hline Stringer free flanges width $\left(w_{1}^{\mathrm{St}}\right)[\mathrm{mm}]$ & 7.6 & 8.5 & 19.1 \\
\hline Stringer bonded flange width $\left(w_{3}^{\mathrm{St}}\right)[\mathrm{mm}]$ & 12.6 & 10.9 & 35.4 \\
\hline Stringer height $\left(w_{4}^{\mathrm{St}}\right)[\mathrm{mm}]$ & 24.2 & 26.2 & 61.9 \\
\hline Stringer thickness $\left(t^{\mathrm{St}}\right)[\mathrm{mm}]$ & 1.4 & 3.2 & 1.8 \\
\hline Skin thickness $\left(t^{\mathrm{Sk}}\right)[\mathrm{mm}]$ & 2.0 & 1.6 & 2.3 \\
\hline Skin-panels count $(n)[-]$ & 28 & 22 & 18 \\
\hline
\end{tabular}

Table 2: Geometrical parameters of the literature solution.

All the components of the structure are considered made of 2024-T3 aluminium alloy, whose mechanical an physical properties have been taken from [31] and are reported in Tab. 3.

\begin{tabular}{llr}
\hline Propriety & Symbol & Value \\
\hline Young's modulus [MPa] & $\mathrm{E}$ & 72395 \\
Poisson's ratio & $\nu$ & 0.33 \\
Tensile yield stress [MPa] & $\sigma_{\mathrm{y}}$ & 290 \\
Tensile ultimate stress $[\mathrm{MPa}]$ & $\sigma_{\mathrm{u}}$ & 434 \\
Density $\left[\mathrm{g} / \mathrm{cm}^{3}\right]$ & $\rho$ & 2.78 \\
\hline
\end{tabular}

Table 3: Material properties of 2024-T3 aluminium alloy.

\subsection{Hypotheses and design criteria}

The case study here presented moves in the framework of the preliminary design phase of aircraft structures. During this phase, tents of load cases (LCs) are assessed to properly design the main components of the structure in order to comply with certification specifications [32]. Such LCs are the result of a combination of basic loading conditions (BLCs) of different nature, e.g. flight loads due to symmetrical manoeuvres, to asymmetrical ones or to gusts, ground loads, pressurisation, 


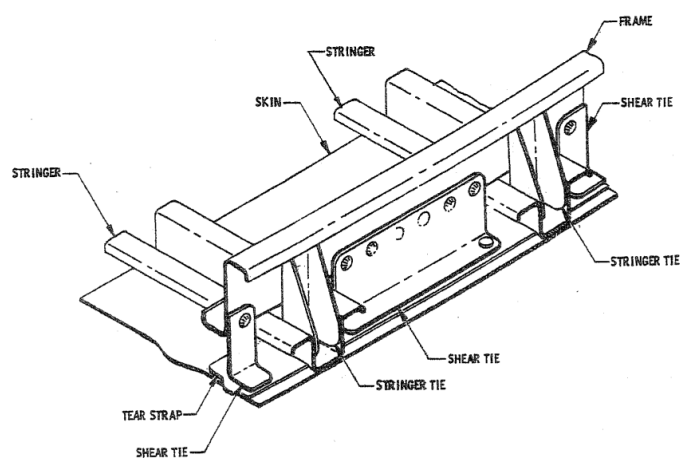

Figure 2: Architecture of the stiffened panel.

etc. In this work, only a sub-set of LCs, presented in Sec. 2.2, is considered. Moreover, for each LC, the material behaviour is supposed linear elastic and the FE analyses are carried out by assuming small displacements and strains.

Concerning the modelling of the structural components, the following simplifications have been introduced:

1. In agreement with the preliminary design framework, only major components of the structure are modelled (i.e. skin, frames, stringers, floor beams and struts).

2. Floor beams and struts have a predefined geometry which is kept unchanged during optimisation.

3. Perfect bonding condition applies at the interface of the structural elements.

4. Connection zones (e.g. floor beams to frames or skin to skin) and opening/cut-out in the skin are not explicitly modelled.

Three main groups of criteria can be identified for the preliminary design phase, i.e. criteria related to: a) static loads, b) fatigue loads and c) aeroelasticity phenomena.

Regarding static loads, certification specifications [32] identify two types of design loads: limit loads (LLs) and ultimate loads (ULs). LLs are the maximum loads expected in service that the structure must be able to support without detrimental permanent deformations. ULs are equal to limit loads multiplied by a prescribed factor of safety (usually 1.5). The structure must withstand ULs without failure for at least 3 seconds. For instance, for the wide-body civil aircraft class, LLs in symmetrical manoeuvres (neglecting gust loads) occur at load factors (the ratio of the aerodynamic force component normal to the longitudinal axis of the aeroplane to its weight) $n_{g}=2.5$ and $n_{g}=-1$. This work focuses on this class of design criteria.

As far as fatigue phenomena are concerned, the design of the structure should be performed in such a way that «catastrophic failure due to fatigue, manufacturing defects, environmental deterioration, or accidental damage, must be avoided throughout the operational life of the aeroplane» [32]. To achieve this goal two approaches are possible. On the one hand, in the framework of the safe-life approach, a component should be designed to last the whole operational life. On the other hand, according to the damage tolerance approach, a potential damage in the structure should not became critical before the next planned inspection. In this work, only one fatigue design criterion following the safe-life approach is employed in terms of an equivalent static check (more details are given in the followings). 
Finally, no aeroelastic criteria are used.

The following set of design criteria (DCs) is integrated in the design process.

DC1 The global stiffness of the structure must be greater than the stiffness of LSol.

DC2 The average equivalent stress in the panels skin must not exceed the yielding stress of the material, considering a factor of safety $\mathrm{F}_{\mathrm{S}}=1.5$ (in agreement with CS 25.303 in [32]), under LLs.

DC3 The average equivalent stress in the skin must not exceed the ultimate strength of the material under ULs.

DC4 No critical fatigue failure must occur caused by the hoop stress in the skin due to pressurisation.

DC5 No buckling must occur in the stiffened panels under ULs (no-buckling design approach).

DC6 Only manufacturable solutions are considered.

DC2 and DC3 are expressed in terms of average stresses in order to neglect the effect of local stress concentrations that could be strongly affected by the accuracy of the FE model and that constitute the object of the detailed design phase (performed after the preliminary design phase).

DC4 is a criterion against the nucleation of cracks in the longitudinal joints between the stiffened panels. It is translated into an equivalent static check according to the methodology reported in $[11,33]$. Such method is based on the definition of the Detail Fatigue Rating (DFR) parameter. It is defined as the maximum stress of a sinusoidal load with a ratio $\mathrm{R}=\sigma_{\min } / \sigma_{\max }=0.06$ producing in $10^{5}$ cycles the same damage of a given fatigue load spectrum. Starting from the knowledge that the maximum hoop stress due to pressurisation of an $\mathrm{ATR}^{\circledR}$ aircraft is $\sigma_{\mathrm{h}}^{\max }=95 \mathrm{MPa}$ for $N=70000$ flights [11], it is possible to compute the equivalent DFR as follows:

$$
\mathrm{DFR}=\frac{\sigma_{\mathrm{mo}} \sigma_{\mathrm{h}}^{\max }(1-\mathrm{R})}{0.53 \sigma_{\mathrm{h}}^{\max }(1-\mathrm{R})+\mathrm{S}^{5-\log N \mathrm{FRF}}\left[0.94 \sigma_{\mathrm{mo}}-0.47 \sigma_{\mathrm{h}}^{\max }(1+\mathrm{R})\right]},
$$

where: $\sigma_{\mathrm{mo}}$ and $\mathrm{S}$ are fatigue material properties that, for a generic aluminium alloy, assume the values $\sigma_{\mathrm{mo}}=310 \mathrm{MPa}$ and $\mathrm{S}=2 ; \mathrm{R}=0$ for the pressurisation cyclic load; FRF is the Fatigue Reliability Factor, i.e. a factor of safety fixed at $\mathrm{FRF}=1.5$.

Under the hypothesis that the required DFR of a structural detail is independent of the aircraft class, one can easily invert Eq. (1) to compute the maximum allowable hoop stress $\sigma_{\mathrm{h}}^{\mathrm{adm}}$. Supposing a target of $N=22500$ flights, typical for a wide-body aircraft, a value of $\sigma_{\mathrm{h}}^{\text {adm }}=126 \mathrm{MPa}$ is obtained.

\subsection{Load cases}

Eight LCs are defined by linear superposition of two BLCs: a cruise loading condition (load factor $n_{g}=1$ ) without pressurisation, identified as $\mathrm{BLC}_{1 \mathrm{~g}}$, and a pressurisation loading condition, identified as $\mathrm{BLC}_{\mathrm{p}}$. In both $\mathrm{BLCs}$, fuselage sections $\mathrm{A}$ and $\mathrm{B}$ are modelled as rigid and $\mathrm{BCs}$ are applied to their centres: section $\mathrm{A}$ is always clamped, whilst pertinent tail forces and moments are applied at section B.

Under $\mathrm{BLC}_{1 \mathrm{~g}}$, payload weight is applied as a distributed load on floor beams. Structural mass is considered by applying additional loads on the upper-deck floor beams, on the basis of statistical estimated structural weight. Tail loads are computed in such a way to obtain in the check zone 
(i.e. the middle bay of the fuselage barrel) a maximum bending moment $M_{\mathrm{x}}=5.0 \cdot 10^{6} \mathrm{Nm}$ and a vertical shear force $F_{\mathrm{y}}=-370000 \mathrm{~N}$ (in agreement with [30]). A good estimation of the loading condition at a different value of the load factor is obtained by scaling $\mathrm{BLC}_{1 \mathrm{~g}}$ by that value.

When using $\mathrm{BLC}_{\mathrm{p}}$, the effect of the maximum operating differential pressure (corresponding to the maximum relief valve setting) is taken into account as internal pressure on the skin plus an equivalent longitudinal force applied to section $\mathrm{B}$ of the fuselage barrel. By scaling $\mathrm{BLC}_{\mathrm{p}}$, the effect of different values of differential pressure can be assessed.

Data used for defining $\mathrm{BLC}_{1 \mathrm{~g}}$ and $\mathrm{BLC}_{\mathrm{p}}$ are reported in Tab. 4. The eight considered LCs are defined in Tab. 5 in which, for each LC, the assessed design criterion is also indicated. Aerodynamics loads on the fuselage have been neglected.

\begin{tabular}{lrr}
\hline Load & $\mathrm{BLC}_{1 \mathrm{~g}}$ & $\mathrm{BLC}_{\mathrm{p}}$ \\
\hline Upper-deck floor beam total load [N] & 10000 & - \\
Lower-deck floor beam total load [N] & 5000 & - \\
Bending moment $M_{\mathrm{x}}$ at section "B" $[\mathrm{Nm}]$ & $4.305 \cdot 10^{6}$ & - \\
Vertical shear force $F_{\mathrm{y}}$ at section "B" [N] & -310000 & - \\
Internal pressure [MPa] & - & 0.068 \\
Longitudinal force $F_{z}[\mathrm{~N}]$ & - & $1.7 \cdot 10^{6}$ \\
\hline${ }^{\dagger}$ Equivalent to internal pressure times fuselage cross-section area.
\end{tabular}

Table 4: Basic loading conditions data.

\begin{tabular}{crrl}
\hline $\mathrm{LC}$ & $\mathrm{BLC}_{1 \mathrm{~g}}$ factor & $\mathrm{BLC}_{\mathrm{p}}$ factor & $\mathrm{DC}$ \\
\hline 1 & 1.00 & 1.00 & $\mathrm{DC} 1$ \\
2 & 2.50 & 1.00 & $\mathrm{DC} 2$ \\
3 & -1.00 & 1.00 & $\mathrm{DC} 2$ \\
4 & 3.75 & 1.00 & $\mathrm{DC} 3$ \\
5 & -1.50 & 1.00 & $\mathrm{DC} 3$ \\
6 & 0 & 1.00 & DC4 \\
7 & 3.75 & 0 & DC5 \\
8 & -1.50 & 0 & DC5 \\
\hline
\end{tabular}

Table 5: Load cases definition and associated design criterion.

\section{Mathematical formulation of the optimisation problem}

\subsection{Design variables}

Only geometrical design variables have been considered in this study. They can be grouped with respect to the component they are referred to.

Stringers and skin. Three circumferential sectors are identified as in Fig. 3: "top", "lateral" and "bottom". For each sector:

- the stringers section is hat-shaped and four variables, $w_{1}^{\mathrm{St}}, w_{3}^{\mathrm{St}}, w_{4}^{\mathrm{St}}$ and $t^{\mathrm{St}}$, are needed to describe its geometry (Fig. 4a);

- the skin is characterised by two variables, i.e. the thickness $t^{\mathrm{Sk}}$ and the number $n$ of sub-regions between two consecutive frames and stringers (hereafter skin-panels) within the sector. 
Frame/shear-tie assembly. Identical frames having a "Z"-shaped cross-section with "L" sheartie are considered: three variables, $w_{1}^{\mathrm{Fr}}, w_{3}^{\mathrm{Fr}}$ and $t^{\mathrm{Fr}}$, are needed to geometrically describe the assembly (Fig. 4b). The distance between the floating frame and the fuselage skin depends on the maximum height of the stringers cross-sections according to the formula

$$
c^{\mathrm{Fr}}=\max _{i} w_{4}^{\mathrm{St}-i}+2 \mathrm{~mm} \quad \text { with } i=\text { Top, Lat, Bot. }
$$

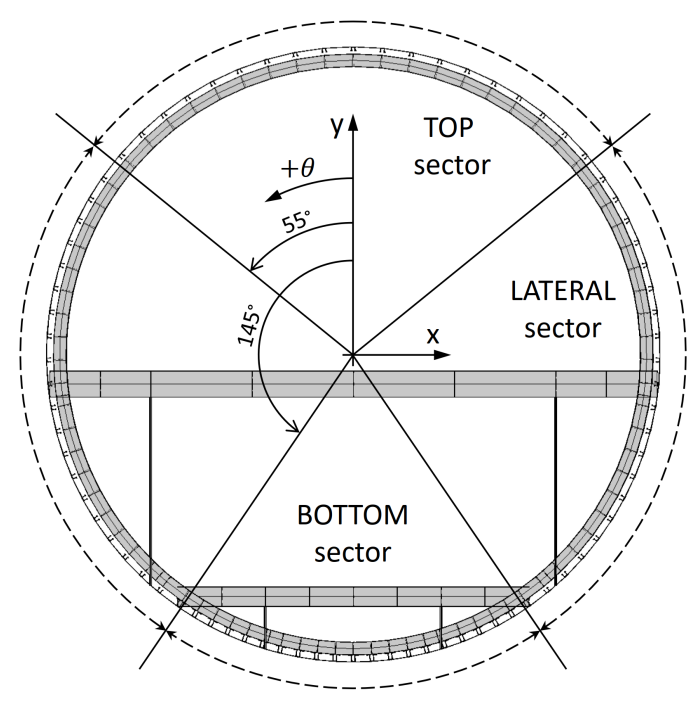

Figure 3: Fuselage cross-section.

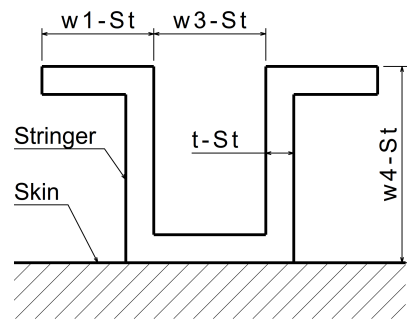

(a) Stringer cross-section

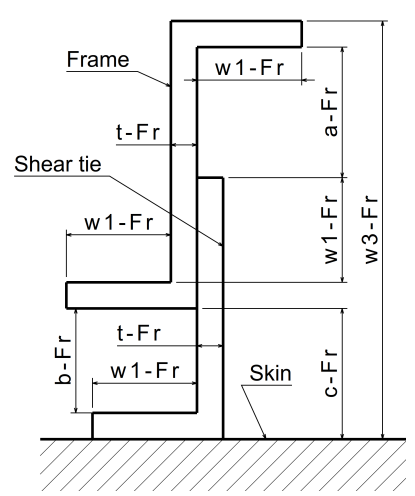

(b) Frame/shear-tie cross-section

Figure 4: Stringers and frame/shear-tie assembly cross-sections variables definition.

All the aforementioned design variables are collected into the vector $\boldsymbol{\xi}$. It is noteworthy that frame pitch, floor beams and struts geometry have not been considered among the problem design variables, rather they have been set equal to the reference values of the LSol as reported in Tabs. 1 and 2 . 


\subsection{Objective and constraint functions}

The goal of the MSO strategy is the minimisation of the total mass of the fuselage barrel which can be easily expressed as

$$
\mathrm{M}(\boldsymbol{\xi})=\mathrm{V}(\boldsymbol{\xi}) \cdot \rho
$$

where $\mathrm{V}(\boldsymbol{\xi})$ is the total volume of the structure components and $\rho$ is the material density as defined in Tab. 3. As far as design requirements are concerned, one or more constraint functions are defined for each DC introduced in Sec. 2.1. In particular, DC1 is formulated as a couple of constraints on the vertical displacement $\delta_{\mathrm{y}}$ and on the rotation $\theta_{\mathrm{x}}$ of the centre of section B when LC1 is considered. These constraints read

$$
\begin{aligned}
& g_{1}(\boldsymbol{\xi})=\left(\delta_{\mathrm{y}}(\boldsymbol{\xi})-\delta_{\mathrm{y}}^{\mathrm{LSol}}\right) / \delta_{\mathrm{y}}^{\mathrm{LSol}} \leq 0, \\
& g_{2}(\boldsymbol{\xi})=\left(\theta_{\mathrm{x}}(\boldsymbol{\xi})-\theta_{\mathrm{x}}^{\mathrm{LSol}}\right) / \theta_{\mathrm{x}}^{\mathrm{LSol}} \leq 0 \quad \text { at } \mathrm{LC} 1 .
\end{aligned}
$$

In Eq. (4), $\delta_{\mathrm{y}}^{\mathrm{LSol}}=-5.21 \mathrm{~mm}$ and $\theta_{\mathrm{x}}^{\mathrm{LSol}}=0.065^{\circ}$ are the vertical displacement and rotation of the centre of section B evaluated for LSol.

DC2 is applied as a set of constraints on the maximum von Mises stress $\left\langle\sigma_{\text {eq }}\right\rangle$ averaged on each skin-panel $\left(\omega_{i}\right)$ belonging to the check zone $\left(\Omega=\sum_{i=1}^{N} \omega_{i}\right.$, see Sec. 4 for more details). Such value has to be lower than the yield stress of the material under LC2 and LC3 with a factor of safety $\mathrm{F}_{\mathrm{S}}=1.5$. Therefore, the related constraint inequalities are:

$$
\begin{aligned}
& g_{3}(\boldsymbol{\xi})=\left(\mathrm{F}_{\mathrm{S}} \cdot \max _{\Omega}\left\langle\sigma_{\mathrm{eq}}(\boldsymbol{\xi})\right\rangle_{\omega_{i}}-\sigma_{\mathrm{y}}\right) / \sigma_{\mathrm{y}} \leq 0 \quad \text { at LC2, } \\
& g_{4}(\boldsymbol{\xi})=\left(\mathrm{F}_{\mathrm{S}} \cdot \max _{\Omega}\left\langle\sigma_{\mathrm{eq}}(\boldsymbol{\xi})\right\rangle_{\omega_{i}}-\sigma_{\mathrm{y}}\right) / \sigma_{\mathrm{y}} \leq 0 \quad \text { at LC3. }
\end{aligned}
$$

DC3 is applied in a similar way to DC2, obtaining:

$$
\begin{aligned}
& g_{5}(\boldsymbol{\xi})=\left(\max _{\Omega}\left\langle\sigma_{\mathrm{eq}}(\boldsymbol{\xi})\right\rangle_{\omega_{i}}-\sigma_{\mathrm{u}}\right) / \sigma_{\mathrm{u}} \leq 0 \quad \text { at LC4 }, \\
& g_{6}(\boldsymbol{\xi})=\left(\max _{\Omega}\left\langle\sigma_{\mathrm{eq}}(\boldsymbol{\xi})\right\rangle_{\omega_{i}}-\sigma_{\mathrm{u}}\right) / \sigma_{\mathrm{u}} \leq 0 \quad \text { at LC5. }
\end{aligned}
$$

As described in Sec. 2.1, DC4 can be formulated as an equivalent constraint on the maximum hoop stress due to pressurisation. Such a requirement can be expressed as follows:

$$
g_{7}(\boldsymbol{\xi})=\left(\max _{\Omega}\left\langle\sigma_{\mathrm{h}}(\boldsymbol{\xi})\right\rangle_{\omega_{i}}-\sigma_{\mathrm{h}}^{\mathrm{adm}}\right) / \sigma_{\mathrm{h}}^{\mathrm{adm}} \leq 0 \quad \text { at LC6 }
$$

The requirement DC5 can be opportunely expressed by means of three optimisation constraints. For each circumferential sector, ULs are applied and the most critical stiffened panel (composed of three stringers and two frames, as discussed in Sec. 4) in the check zone is identified. An eigenvalue buckling analysis is then performed on this panel in order to get a first buckling eigenvalue higher than 1 with a factor of safety $F_{S}=1.1$ (more details on this point are given in Sec. 4). The related 
constraints read

$$
\begin{array}{ll}
g_{8}(\boldsymbol{\xi})=1.1-\lambda^{\text {Top }}(\boldsymbol{\xi}) \leq 0 & \text { at LC8 } \\
g_{9}(\boldsymbol{\xi})=1.1-\lambda^{\text {Lat }}(\boldsymbol{\xi}) \leq 0 & \text { at LC7 } \\
g_{10}(\boldsymbol{\xi})=1.1-\lambda^{\text {Bot }}(\boldsymbol{\xi}) \leq 0 & \text { at LC7 }
\end{array}
$$

DC6 is applied by imposing a series of inequalities involving the design variables and representing different types of manufacturability requirements.

- Minimum thickness of thin-walled elements.

$$
\begin{aligned}
& t^{\mathrm{St}-i} \geq 1 \mathrm{~mm}, \quad \text { with } i=\text { Top, Lat, Bot; } \\
& t^{\mathrm{Sk}-i} \geq 1 \mathrm{~mm}, \quad \text { with } i=\text { Top, Lat, Bot; } \\
& t^{\mathrm{Fr}} \geq 1 \mathrm{~mm} .
\end{aligned}
$$

- Minimum length of the interface flange of stiffening components for the installation of rivets.

$$
\begin{aligned}
& w_{3}^{\text {St-i }} \geq 14 \mathrm{~mm}, \quad \text { with } i=\text { Top, Lat, Bot; } \\
& w_{1}^{\mathrm{Fr}} \geq 14 \mathrm{~mm}
\end{aligned}
$$

- Minimum length to thickness ratio of sheet elements.

$$
\begin{array}{ll}
w_{1}^{\mathrm{St}-i} / t^{\mathrm{St}-i} \geq 4, & \text { with } i=\text { Top, Lat, Bot; } \\
w_{3}^{\mathrm{St}-i} / t^{\mathrm{St}-i} \geq 3, & \text { with } i=\text { Top, Lat, Bot; } \\
w_{4}^{\mathrm{St}-i} / t^{\mathrm{St}-i} \geq 5, & \text { with } i=\text { Top, Lat, Bot; } \\
w_{1}^{\mathrm{Fr}} / t^{\mathrm{Fr}} \geq 3 ; & \\
a^{\mathrm{Fr}}(\boldsymbol{\xi}) / t^{\mathrm{Fr}} \geq 3 ; & \\
b^{\mathrm{Fr}}(\boldsymbol{\xi}) / t^{\mathrm{Fr}} \geq 3 . &
\end{array}
$$

- Minimum circumferential distance between stringers.

$$
\operatorname{pitch}^{\mathrm{St}-i}(\boldsymbol{\xi}) \geq 2 \cdot\left(2 \cdot w_{1}^{\mathrm{St}-i}+w_{3}^{\mathrm{St}-i}\right), \quad \text { with } i=\text { Top, Lat, Bot. }
$$

These constraints behave more like logic conditions (i.e. True / False values) rather than continuousvalued functions. Inasmuch as these constraint functions are mainly related to Boolean operations, it is sufficient that each of them is satisfied regardless of its value, i.e. a value closer to the bounds would not produce any advantage.

Some of these inequalities are directly employed in the definition of the lower and upper bounds of the design variables for the problem at hand, as listed in Tab. 6, whilst the remaining inequalities are stated in the form $l_{j}(\boldsymbol{\xi}) \leq 0$ and then aggregated into a single constraint using the maximum operator:

$$
g_{11}(\boldsymbol{\xi})=\max _{j} l_{j}(\boldsymbol{\xi}) \leq 0
$$


When a completely unfeasible solution is detected (e.g. a solution with an overlap between stringers), the corresponding FE models are not generated and the solution is penalised by assigning a high value to the objective function and a unit value to the constraints that cannot be evaluated.

It is noteworthy that the step size of the the thickness of the different components has been fixed to $\Delta t=0.1 \mathrm{~mm}$ to be coherent with the thickness of commercially available aluminium sheets.

Finally, the optimisation problem can be formulated as a classical CNLPP as follows:

$$
\begin{aligned}
& \min _{\boldsymbol{\xi}} \mathrm{M}(\boldsymbol{\xi}), \\
& \text { subject to: } \\
& g_{i}(\boldsymbol{\xi}) \leq 0, \quad \text { with } i=1,2, \ldots, 11 .
\end{aligned}
$$

The design space of the problem is detailed in Table 6 .

\begin{tabular}{lcrrr}
\hline Design variable & Unit & Lower bound & Upper bound & Step size \\
\hline$w_{1}^{\mathrm{Fr}}$ & $\mathrm{mm}$ & 20 & 50 & 0.5 \\
$w_{3}^{\mathrm{Fr}}$ & $\mathrm{mm}$ & 80 & 190 & 1.0 \\
$t^{\mathrm{Fr}}$ & $\mathrm{mm}$ & 1 & 4 & 0.1 \\
$w_{1}^{\mathrm{St}-i}$ & $\mathrm{~mm}$ & 5 & 30 & 0.5 \\
$w_{3}^{\mathrm{St}-i}$ & $\mathrm{~mm}$ & 14 & 40 & 0.5 \\
$w_{4}^{\mathrm{St}-i}$ & $\mathrm{~mm}$ & 14 & 70 & 0.5 \\
$t^{\mathrm{St}-i}$ & $\mathrm{~mm}$ & 1 & 4 & 0.1 \\
$t^{\mathrm{Sk}-i}$ & $\mathrm{~mm}$ & 1 & 4 & 0.1 \\
$n^{\mathrm{Top}}$ & - & 18 & 38 & 2 \\
$n^{\mathrm{Lat}}$ & - & 13 & 31 & 1 \\
$n^{\mathrm{Bot}}$ & - & 12 & 26 & 2 \\
\hline With $i=$ Top, Lat, Bot. & & &
\end{tabular}

Table 6: Lower and upper bounds of the design variables.

\subsection{Numerical strategy}

Problem (10) is a non-convex CNLPP. The total number of design variables is 21, whilst the number of optimisation constraints is 11. For the resolution of problem (10) the GA ERASMUS [29] coupled with both GFEM and LFEMs of the structure has been utilised as optimisation tool to perform the solution search, as illustrated in Fig. 5. The GA ERASMUS has already been successfully applied to solve different kinds of engineering problems, see for example [29, 34-41].

As shown in Fig. 5, for each individual, at each generation, the numerical tool performs global and local FE analyses to calculate the objective function and the optimisation constraints. The FE models are implemented in the ANSYS ${ }^{\circledR}$ environment and their input data are generated by the GA ERASMUS (more details are given in Sec. 4). The GA elaborates the results provided by the GFEM and the LFEMs in order to execute the genetic operations and generate new individuals. These operations are repeated until the GA meets the user-defined convergence criterion. The generic individual of the GA ERASMUS represents a potential solution for the problem at hand. The genotype of the individual for problem (10) is characterised by only one chromosome composed of 21 genes, each one coding a component of the vector of design variables $\boldsymbol{\xi}$. 


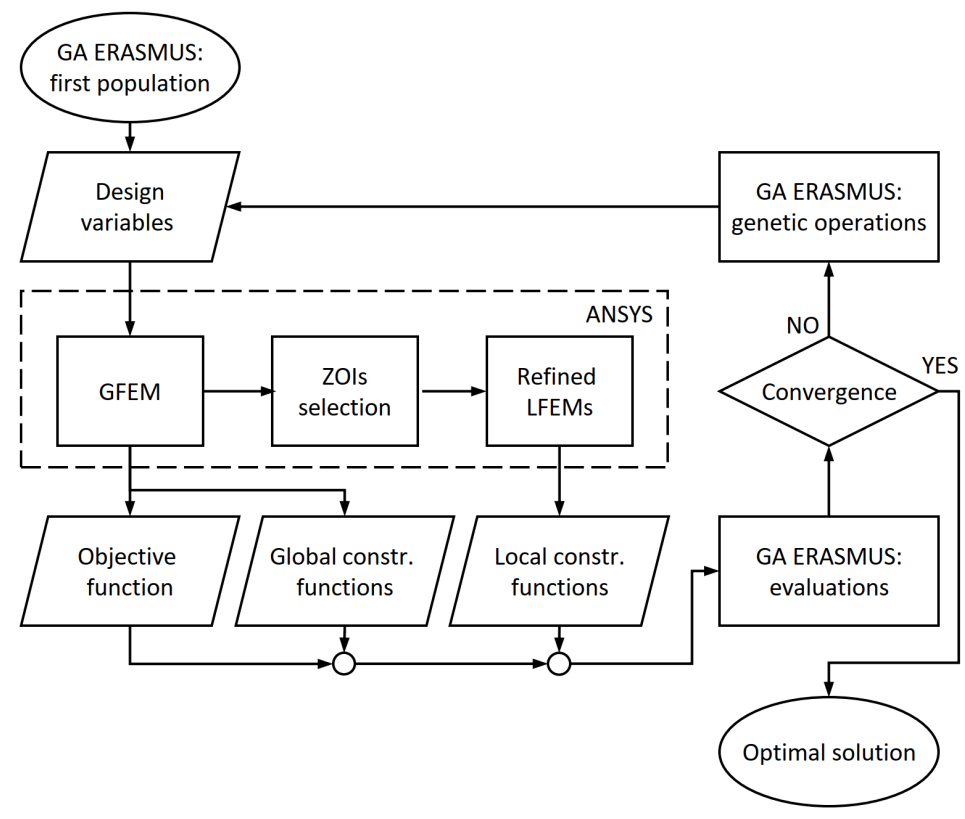

Figure 5: Flowchart of the optimisation process.

\section{The global/local finite element modelling approach}

As stated above, the FE models integrated in the optimisation process are based on a GL modelling approach. In particular, two different models are created: the GFEM for the assessment of the behaviour of the whole fuselage barrel, and refined LFEMs in order to properly evaluate local responses. LFEMs are created only at the critical ZOIs identified during the global analysis, thus suitable criteria must be developed to accomplish this task.

Both GFEM and LFEMs are fully parametric and are built using the commercial FE code ANSYS $^{\circledR}$.

\subsection{The global finite element model}

The global FE model is shown in Fig. 6: it includes the seven bays constituting the fuselage barrel. The fuselage skin is modelled with 8-node SHELL281 elements, while frames, stringer, floor beams and struts are modelled with 3-node BEAM189 elements. The beam and shell elements are connected together by node merging. To take into account the actual position of the beam cross-section with respect to the skin, a section offset is applied to beam elements. Shear-tie components are not modelled, but their mechanical effect (the transfer of shear load from the frames to the skin) is ensured by the direct connection between frame and skin elements.

The element type (linear or quadratic) and mesh size have been chosen after performing a sensitivity analysis, of which the main details can be found in Appendix A. In particular, the use of one quadratic element for each skin-panel gives a good compromise between results accuracy and computational cost, thus the mesh size has been set accordingly.

A master node is created at the centre of sections A and B and linked to the set of "slave" nodes of the corresponding frame by means of MPC184 (multi-point constraint) elements with "rigid beam" behaviour (Fig. 6a). These master nodes are used to apply the BCs presented in Sec. 2.2. In agreement with the hypotheses and the design criteria discussed in Sec. 2.1, only linear static analyses are performed on this model. 


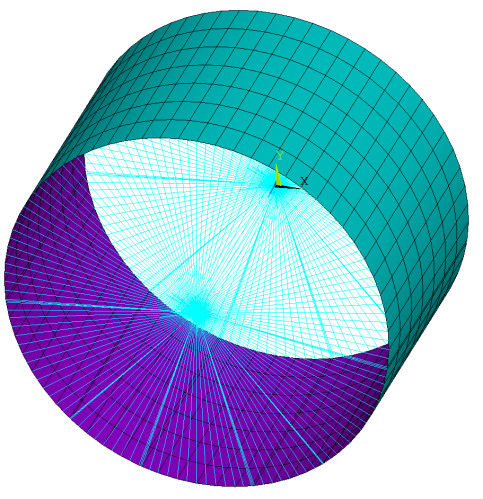

(a) Shell and MPC elements

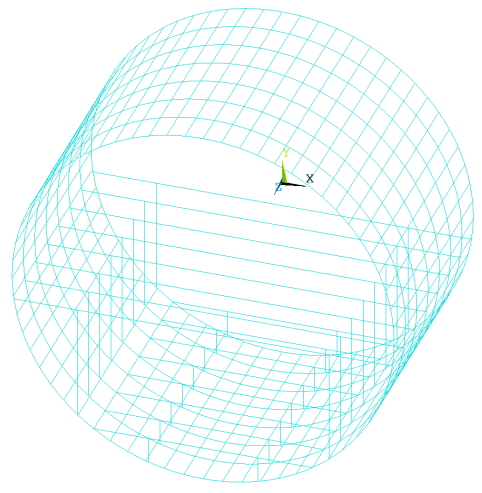

(b) Beam elements

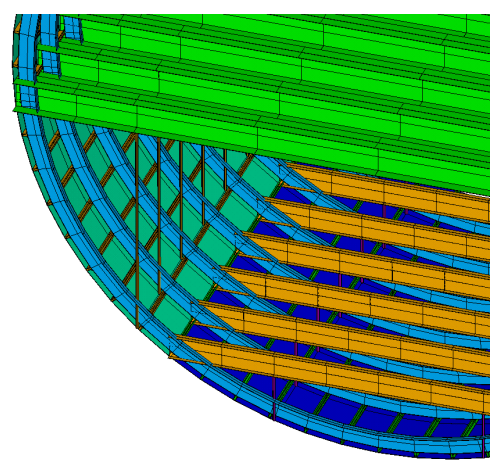

(c) Detailed view with the integral shape of the elements

Figure 6: Global FE model.

Of course, the first bay (from each side of the fuselage barrel) is strongly influenced by edge effects because of the proximity to zones where BCs are applied (i.e. at nodes A and B). Accordingly, only the central bay constitutes the check zone, where the results of the analysis are meaningful. Moreover, as explained in Sec. 2.1, the elements adjacent to connection zones (e.g. floor beams to frames connections or the joints between circumferential sectors) are excluded from the check zone, as illustrated in Fig. 7.

Results provided by the GFEM are used for the evaluation of the objective function and all the constraint functions except those related to buckling requirements, i.e. $g_{8}, g_{9}$ and $g_{10}$.

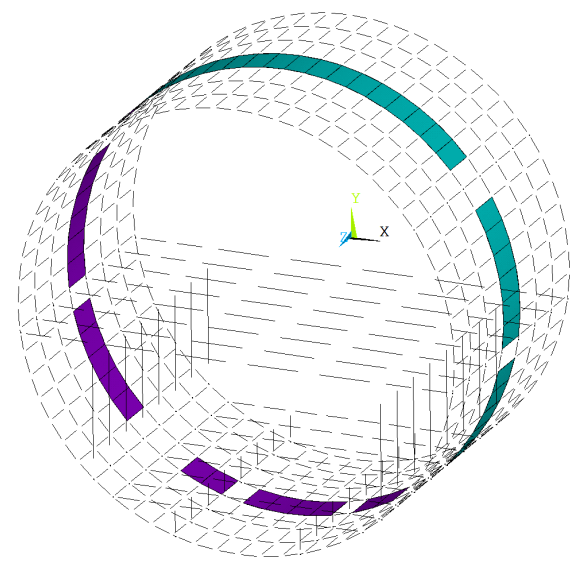

Figure 7: Check zone of the global FE model.

\subsection{The local finite element models}

LFEMs are created to evaluate the first buckling load of the most critical fuselage stiffened panels. This task can be achieved only through a suitably refined FE model able to catch both global and local buckling modes.

Each LFEM includes the same number of stringers and frames, i.e. three and two, respectively, as shown in Fig. 8. The local model presents a suitable extinction zone to mitigate edge effects due to the application of BCs. This extinction zone is half a skin-panel wide and surrounds the 
check zone, as illustrated in Fig 8. The local FE model is entirely built by using 8-node SHELL281 elements.

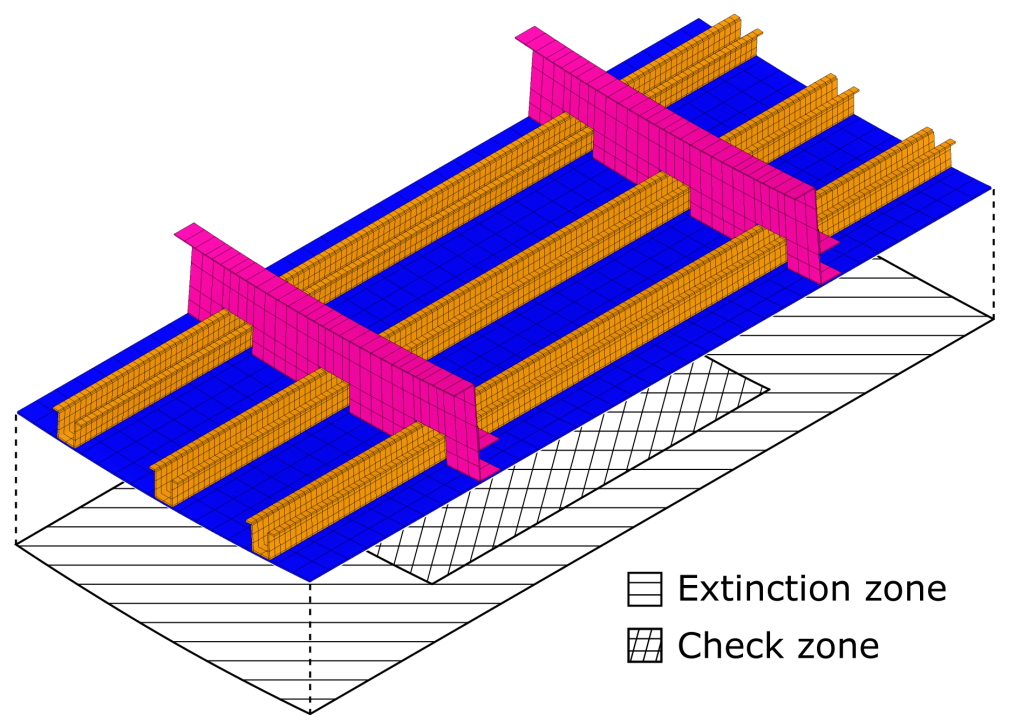

Figure 8: Typical local FE model.

Both shear-ties and stringers are tied to the skin by creating constraint equations between their interface nodes by using ANSYS ${ }^{\circledR}$ CEINTF command [42]. This allows having an independent mesh size on the different components. Also for the LFEM, the mesh size is the result of a compromise between the accuracy in evaluating the first buckling load of the stiffened panel, which can occur either in the skin or in the flanges of stringers and frames, and the computational time. A sensitivity analysis has been conducted also in this case: the main results are reported in Appendix A.

Displacement BCs extracted from the results of the global analysis are imposed to all the boundary nodes belonging to the skin of the local FE model.

To transfer the BCs to the stringers and frames, for each ending cross-section, a master node is extracted from the skin boundary nodes located at the interface between the beam reference axis and the skin in the GFEM. The coordinates of this set of master nodes are recorded and passed to the LFEM (for each region of the fuselage barrel). Then these nodes are selected and connected to those belonging to the corresponding stringer/frame ending cross-section by means of MPC184 elements with "rigid beam" behaviour, ensuring in this way the kinematic compatibility between global and local models, see Fig. 9.
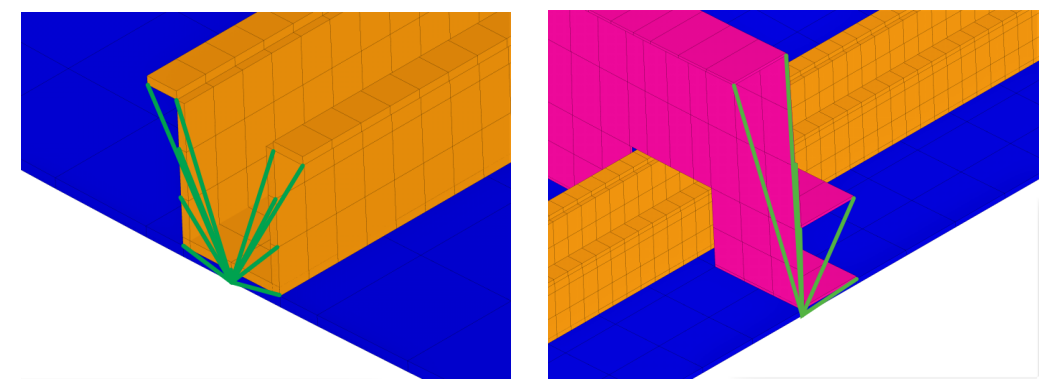

Figure 9: Detail of the ending cross-section of the stiffening components in the local FE model.

The LFEM is built for each sector of the fuselage barrel (bottom, top and lateral). An eigenvalue 
buckling analysis is performed on the local models, and the lowest positive eigenvalue, $\lambda(\boldsymbol{\xi})$, is retrieved as output.

\subsection{ZOIs identification criteria and information transfer between global and local models}

In the presented MSO strategy, the fewest number of local models is checked in order to keep the computational time as low as possible. To this purpose, specific criteria have been introduced and applied to the post-processing of results coming from the GFEM in order to identify the most critical ZOIs around which LFEMs are automatically generated. For each circular sector belonging to the check zone, only one ZOI is identified and analysed.

As discussed in Secs. 2.2 and 3.2, the buckling-related constraints are evaluated for LC7 and LC8 (see Tab. 5). These LCs are obtained by scaling $\mathrm{BLC}_{1 \mathrm{~g}}$ by means of a suitable load factor. Under $\mathrm{BLC}_{1 \mathrm{~g}}$, the stiffened panels in the top and bottom sectors are mostly subject to stress in the longitudinal direction, as shown in Fig. 10. Therefore, top and bottom ZOIs are identified by

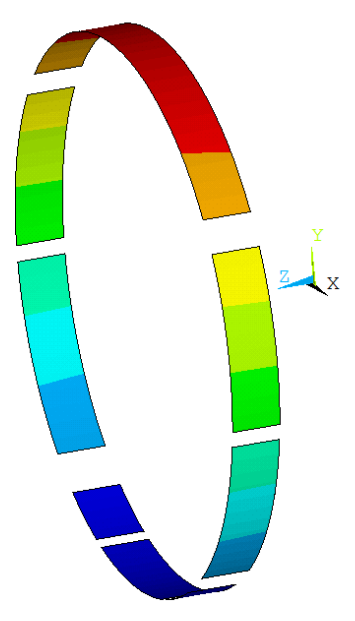

(a) Longitudinal stress

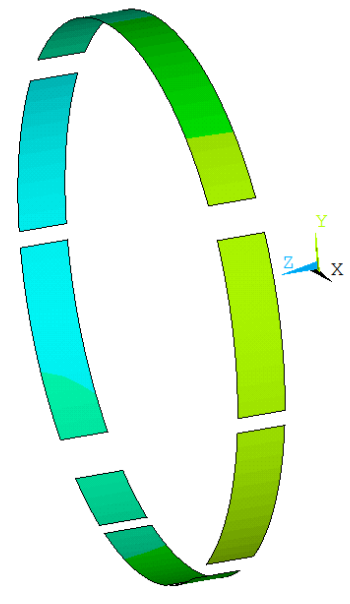

(b) Shear stress

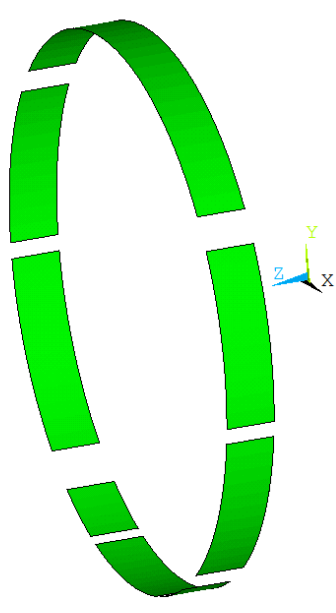

(c) Hoop stress

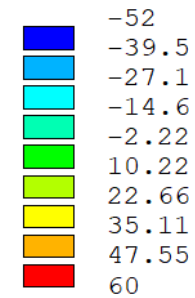

(d) Legend $[\mathrm{MPa}]$

Figure 10: Stress distribution in the skin-panels in the check zone at $\mathrm{BLC}_{1 \mathrm{~g}}$.

looking for the basic-panel (BP), i.e. the assembly composed of a stringer plus half of the adjacent skin-panels, that withstands the highest compressive average longitudinal force per unit width, $\mathrm{N}_{1}^{\mathrm{BP}}$, computed, for the generic LC, as

$$
\mathrm{N}_{\mathrm{l}}^{\mathrm{BP}}=\frac{\mathrm{F}^{\mathrm{st}}+\int_{-w^{\mathrm{sp}} / 2}^{w^{\mathrm{sp}} / 2} \int_{0}^{t^{\mathrm{sk}}} \sigma_{1}^{\mathrm{sk}} \mathrm{d} z \mathrm{~d} y}{w^{\mathrm{sp}}}
$$

where: $\mathrm{F}^{\text {st }}$ is the axial tensile force in the stringer, $w^{\mathrm{sp}}$ is the width, in the hoop direction, of the skin panel and $\sigma_{1}^{\text {sk }}$ is the longitudinal stress in the skin.

On the other hand, the panels in the lateral sector are subject to biaxial loads corresponding to a combination of mainly shear and longitudinal stress; the latter varying from tensile to compression depending on the position of the considered stiffened panel (see Fig. 10). Accordingly, a different criterion is used for the lateral sector: the ZOI is identified by looking for the most critical skinpanel with respect to the buckling strength. An estimation of the buckling load is computed for each skin-panel in the check zone using analytical formulae for a simply supported "shadow" plate with the same dimensions of the analysed skin-panel, i.e. $a$ in the longitudinal direction and $b$ in the hoop one,the same thickness $t^{\text {sk }}$ and subject to the same bi-axial stress field given by the membrane 
forces per unit length $\mathrm{N}_{\mathrm{x}}, \mathrm{N}_{\mathrm{y}}$, and $\mathrm{N}_{\mathrm{xy}}$. The buckling factor due to bi-axial compression forces and that due to shear forces are computed independently and an interaction equation is then used to compute the buckling factor due to the combined load. Under the hypothesis that the plate buckles with an out-of-plane displacement field described by

$$
w(x, y)=\sum_{m=1}^{\infty} \sum_{n=1}^{\infty} a_{m n} \sin \frac{m \pi x}{a} \sin \frac{n \pi y}{b},
$$

the buckling eigenvalue due to the solely compressive membrane forces per unit length $\mathrm{N}_{\mathrm{x}}$ and $\mathrm{N}_{\mathrm{y}}$, can be computed as [43]

$$
\lambda^{\mathrm{c}}=\min _{m, n} \pi^{2} \frac{\mathrm{D}\left[(m / a)^{2}+(n / b)^{2}\right]^{2}}{\mathrm{~N}_{\mathrm{x}}(m / a)^{2}+\mathrm{N}_{\mathrm{y}}(n / b)^{2}}
$$

where $\mathrm{x}$ and $\mathrm{y}$ directions correspond to longitudinal and hoop ones and $\mathrm{D}$ is the flexural rigidity of the plate. The buckling eigenvalue due to the solely shear forces per unit width $\mathrm{N}_{\mathrm{xy}}$ can be approximated as [43]

$$
\lambda^{\mathrm{s}} \approx \frac{\left(5.35+4 r^{2}\right) \pi^{2} \mathrm{D}}{\mathrm{N}_{\mathrm{xy}} c^{2}}
$$

where: $r=\min (a / b, b / a)$ and $c=\min (a, b)$. An interaction equation can be used to define the critical condition in presence of the two loading conditions [44]

$$
\left(\frac{1}{\lambda_{\mathrm{cr}}^{\mathrm{s}}}\right)^{2}+\frac{1}{\lambda_{\mathrm{cr}}^{\mathrm{c}}}=1
$$

If the critical buckling factor, for the combined loading conditions, is defined as

$$
\lambda_{\mathrm{cr}}:=\frac{\lambda^{\mathrm{s}}}{\lambda_{\mathrm{cr}}^{\mathrm{s}}}=\frac{\lambda^{\mathrm{c}}}{\lambda_{\mathrm{cr}}^{\mathrm{c}}}
$$

one can calculate its value according to the following formula:

$$
\lambda_{\mathrm{cr}}=\frac{-\frac{\lambda^{\mathrm{s} 2}}{\lambda^{\mathrm{c}}}+\sqrt{\left(\frac{\lambda^{\mathrm{s} 2}}{\lambda^{\mathrm{c}}}\right)^{2}+4 \lambda^{\mathrm{s}^{2}}}}{2}
$$

Therefore, the skin-panel showing the minimum $\lambda_{\text {cr }}$ identifies the lateral ZOI.

As already stated, the displacement field resulting from the GFEM is used to define the BCs for the LFEMs. To this purpose, for each LC, the nodal displacements of the GFEM are interpolated using the shape functions of the elements in the GFEM at the location of the boundary nodes of the LFEM. The logical flow of the process that goes from the global FE analysis to the local one is given in Fig. 11.

\section{Numerical results}

The parameters of the GA ERASMUS used to perform the solution search for problem (10) are listed in Tab. 7. As far as the optimisation constraints are concerned, they have been han- 


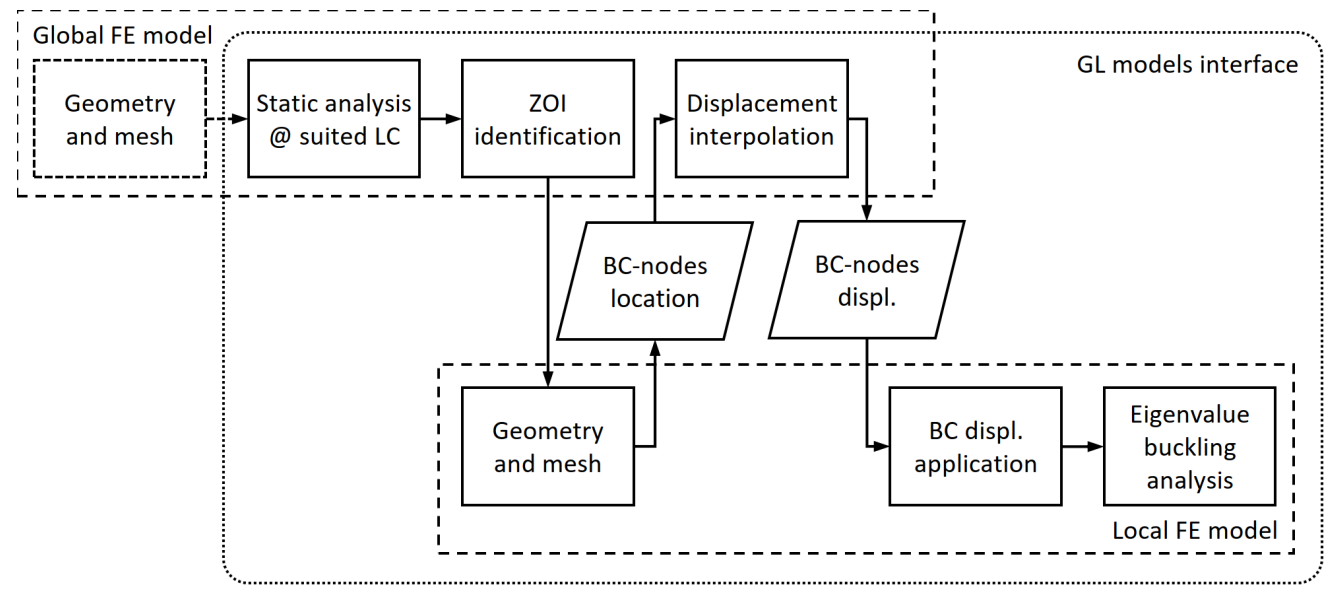

Figure 11: Interaction scheme between global and local finite element models.

dled through the Automatic Dynamic Penalisation (ADP) method [45]. Further details on the optimisation tool and its parameters can be found in [29].

\begin{tabular}{lr}
\hline Property & Value \\
\hline N. of populations & 1 \\
N. of individuals per population & 210 \\
N. of chromosomes & 1 \\
N. of genes & 21 \\
Stop criterion & Fixed generations $(150)$ \\
Crossover probability & 0.85 \\
Mutation probability & 0.01 \\
Selection operator & Roulette wheel \\
Fitness pressure & 1 \\
Elitism operator & Active \\
\hline
\end{tabular}

Table 7: Parameters of the GA ERASMUS used for the solution search.

The whole optimisation process requires a computational time of approximately 14 days (i.e. around $40 \mathrm{~s}$ for global and local FE analyses for the generic point in the design space) when four cores of a machine with an Intel Xeon E5-2697v2 processor $(2.70-3.50 \mathrm{GHz})$ are dedicated to the ANSYS ${ }^{\circledR}$ solver. However, computational time could be easily reduced by performing in parallel the FE calculations of the different individuals.

The evolution of the objective function of the best individual for each generation of the optimisation process is shown in Fig. 12. It can be noticed that the convergence is achieved after about 100 generations because no improvement in the objective function is observed in the subsequent iterations of the evolutionary process. From Fig. 12 three stages can be identified corresponding to first, intermediate and last generations, i.e. stages A, B and C, respectively. The complete set of performances, in terms of constraint and objective functions, for the best individual at each stage, i.e. individuals A-1, B-1 and C-1, is reported in Tab. 8. Concerning stage C, two other individuals (C-2 and C-3) are reported together with the optimum solution C-1 (recall that the CNLPP is strongly non-convex). Indeed, a significant number of pseudo-optimal solutions exists at stage $\mathrm{C}$ that are nearly identical to solution $\mathrm{C}-1$ and that are not reported here for the sake of brevity. The values of the design variables identifying the individuals C-1, C-2 and C-3 are listed in Tab. 9.

A quick glance to the results provided in Tabs. 8 and 9 suffices to infer that, due the non-convex nature of problem (10), the GA finds almost equivalent optimal solutions, e.g. C-1, C-2 and C-3, 


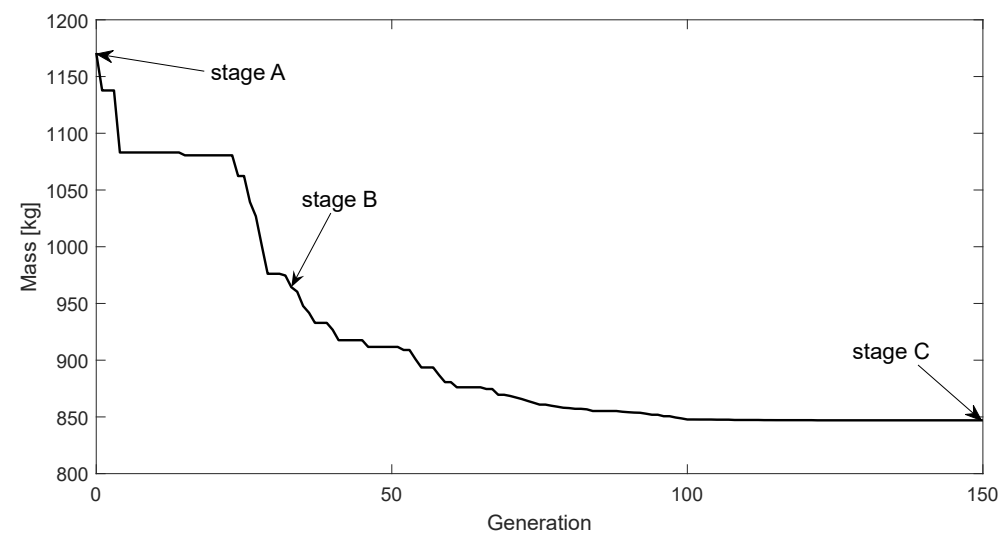

Figure 12: Best individual vs. iterations.

\begin{tabular}{|c|c|c|c|c|c|c|c|}
\hline \multicolumn{2}{|c|}{ Function } & \multirow{2}{*}{$\begin{array}{r}\text { A-1 } \\
-0.467\end{array}$} & \multirow{2}{*}{$\begin{array}{r}\mathrm{B}-1 \\
-0.331\end{array}$} & \multirow{2}{*}{$\begin{array}{r}\text { C-3 } \\
-0.227\end{array}$} & \multirow{2}{*}{$\begin{array}{r}\mathrm{C}-2 \\
-0.238\end{array}$} & \multirow{2}{*}{$\frac{\mathrm{C}-1^{*}}{-0.262}$} & \multirow{2}{*}{$\begin{array}{r}\text { LSol } \\
-0.000\end{array}$} \\
\hline$g_{1}$ & (stiffness) & & & & & & \\
\hline$g_{2}$ & (stiffness) & -0.426 & -0.115 & -0.190 & -0.179 & -0.053 & -0.000 \\
\hline$g_{3}$ & (strength) & -0.446 & -0.076 & -0.163 & -0.179 & -0.013 & +0.020 \\
\hline$g_{4}$ & (strength) & -0.683 & -0.479 & -0.573 & -0.573 & -0.426 & -0.487 \\
\hline$g_{5}$ & (strength) & -0.642 & -0.420 & -0.466 & -0.477 & -0.378 & -0.350 \\
\hline$g_{6}$ & (strength) & -0.822 & -0.718 & -0.759 & -0.760 & -0.689 & -0.726 \\
\hline$g_{7}$ & (fatigue) & -0.578 & -0.239 & -0.357 & -0.387 & -0.197 & -0.213 \\
\hline$g_{8}$ & (buckling) & -1.845 & -0.155 & -0.133 & -0.110 & -0.070 & +0.535 \\
\hline$g_{9}$ & (buckling) & -0.513 & -0.034 & -0.191 & -0.087 & -0.029 & +0.811 \\
\hline$g_{10}$ & (buckling) & -0.484 & -0.234 & -0.001 & -0.213 & -0.027 & +0.570 \\
\hline$g_{11}$ & (manufacturability) & -0.024 & -0.030 & -0.560 & -0.033 & -0.545 & +1.344 \\
\hline M & {$[\mathrm{kg}]$} & 1158 & 966 & 886 & 871 & 847 & 837 \\
\hline
\end{tabular}

Table 8: Comparison of the values of the constraint and objective functions relative to different individuals.

quite different in term of design variables, that still respect all the constraints and that have comparable values of the objective function.

Concerning the retained optimal solution, i.e. C-1, from Tab. 9 one can notice that some of the variables are located at the bounds of the respective intervals, see Tab. 6 .

By observing the buckling mode of each one of the three optimised sectors for solution C-1, as illustrated in Fig. 13, it can be noticed that the stringers and the skin buckle simultaneously, in agreement with the well-established aeronautical design criterion that the maximum structural efficiency (in terms of best compromise between minimum weight and maximum buckling load) for stiffened structures is reached when their components buckle at the same load [46].

Finally, for comparison purposes, the performances of LSol and some of its geometrical parameters, previously shown in Tab. 2, are also added in Tabs. 8 and 9, respectively. From the analysis of the results, it is clear that LSol has been obtained with different requirements than those used in this work; in particular, constraints $g_{3}, g_{8}, g_{9}$ and $g_{10}$ are too restrictive for LSol, which results clearly infeasible. Also the geometrical requirement $g_{11}$ is not respected by LSol which is characterised by too close stringers in the bottom sector. Moreover, it is noteworthy that, despite of the big differences in performances, the difference in mass between C-1 and LSol is lower than 1.2\%. Therefore, the proposed approach allows finding an optimal configuration of the fuselage barrel satisfying the full set of design requirements. This result constitutes a sort of "numerical proof" 


\begin{tabular}{|c|c|c|c|c|c|c|c|c|c|c|c|c|}
\hline Variable & Top & $\begin{array}{c}\text { C-3 } \\
\text { Lat. }\end{array}$ & Bot. & Top & $\begin{array}{c}\text { C-2 } \\
\text { Lat. }\end{array}$ & Bot. & Top & $\begin{array}{c}\text { C-1* } \\
\text { Lat. }\end{array}$ & Bot. & Top & $\begin{array}{l}\text { LSol } \\
\text { Lat. }\end{array}$ & Bot. \\
\hline$w_{1}^{\mathrm{Fr}}[\mathrm{mm}]$ & & 28.5 & & & 29.0 & & & $20.0^{\dagger}$ & & & 35.0 & \\
\hline$w_{3}^{\mathrm{Fr}}[\mathrm{mm}]$ & & 116.5 & & & 120.0 & & & 81.0 & & & 165.0 & \\
\hline$t^{\mathrm{Fr}}[\mathrm{mm}]$ & & 1.2 & & & 1.2 & & & $1.0^{\dagger}$ & & & 1.5 & \\
\hline$w_{1}^{\mathrm{St}}[\mathrm{mm}]$ & 6.0 & 8.0 & 17.5 & 6.5 & 7.5 & 18.5 & $5.0^{\dagger}$ & $5.0^{\dagger}$ & 21.0 & 7.6 & 8.5 & 19.1 \\
\hline$w_{3}^{\mathrm{St}}[\mathrm{mm}]$ & 23.0 & 27.5 & 26.0 & 25.5 & 27.0 & 28.0 & $40.0^{\dagger}$ & 24.5 & 31.0 & 12.6 & 10.9 & 35.4 \\
\hline$w_{4}^{\mathrm{St}}[\mathrm{mm}]$ & 23.5 & 33.0 & 64.5 & 27.5 & 21.5 & 61.5 & 19.0 & 38.0 & 45.5 & 24.2 & 26.2 & 61.9 \\
\hline$t^{\mathrm{St}}[\mathrm{mm}]$ & 1.3 & 1.2 & 2.5 & 1.6 & 1.4 & 1.6 & 1.1 & $1.0^{\dagger}$ & 1.4 & 1.4 & 3.2 & 1.8 \\
\hline$t^{\mathrm{Sk}}[\mathrm{mm}]$ & 2.5 & 2.2 & 2.5 & 2.5 & 2.3 & 2.4 & 1.8 & 3.0 & 3.6 & 2.0 & 1.6 & 2.3 \\
\hline$n[-]$ & 30 & 30 & 22 & 28 & 30 & $26^{\dagger}$ & 36 & 24 & 18 & 28 & 22 & 18 \\
\hline
\end{tabular}

Table 9: Design variables values for optimal individuals at the last generation.

about the limitations related to the use of simplifying hypotheses and analytical formulae/models in the framework of the preliminary design phase of aircraft structures.

\section{Conclusions}

A multi-scale optimisation strategy for designing thin-walled structures integrating a dedicated global-local modelling approach has been presented in this work.

As usual for this type of structures, the design problem is formulated as a CNLPP involving constraints of different nature. The formulation of such requirements involves the structure response, at each pertinent scale, under various loading conditions. To deal with these aspects, fully parametric FE models are created at different scales for the evaluation of the most relevant phenomena. A coherent information transfer between these models is ensured by implementing a sub-modelling GL approach: BCs of the local models are directly extracted, in terms of displacements, from the results of the global analysis and properly transferred to the local FE model. Local FE models are automatically created only for critical ZOIs which are identified, by means of opportune criteria, during the global analysis. The solution search for the multi-scale CNLPP is performed by interfacing the GFEM and the LFEMs of the structure with the GA ERASMUS developed at the I2M laboratory in Bordeaux.

The proposed strategy is general and allows dealing with design variables and constraints of different nature. Every variable at each relevant scale is considered in the design process, avoiding the introduction of simplifying hypotheses in the definition of the design space, which have the main effect of shrinking it, thus preventing the possibility to find a true global optimum solution. Moreover, by employing a GL modelling approach, more accurate results are obtained than those found by means of well-established strategies that use simplified analytical models for the assessment of the mechanical response of the structure, as proven by the numerical results of this study. Finally, the whole process, once set, is fully automated and does not need the user intervention.

The effectiveness of the proposed MSO strategy is proven on a meaningful design case: the least-weight design of an aluminium fuselage barrel of a wide-body aircraft. In the considered test case, a limited, yet representative, set of loading conditions and design criteria are considered. Nevertheless, further criteria and load cases could be easily introduced in the general framework of the presented design strategy. 


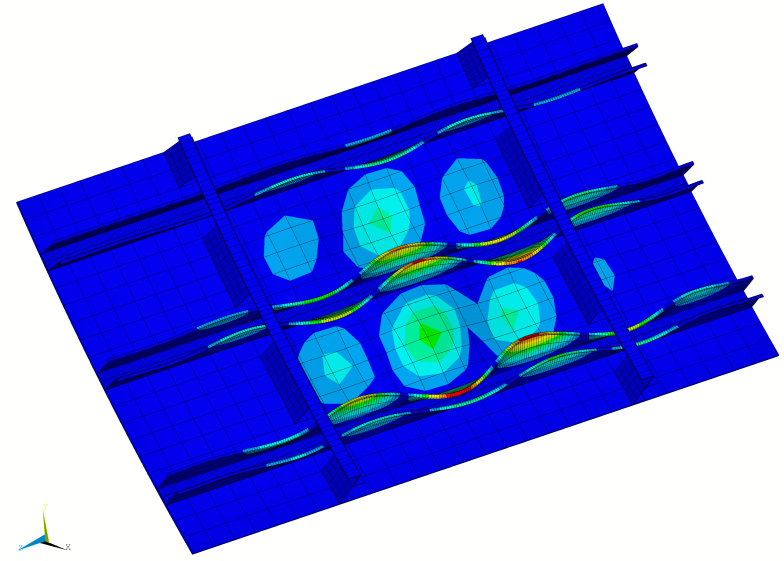

(a) Lateral sector panel

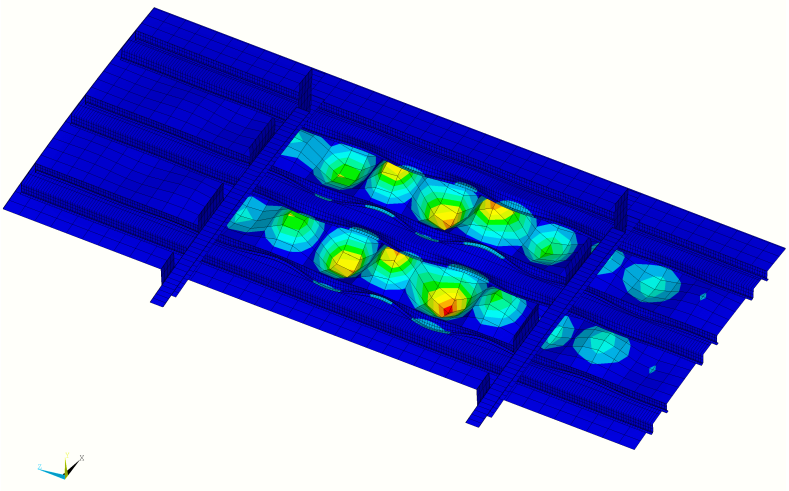

(b) Top sector panel

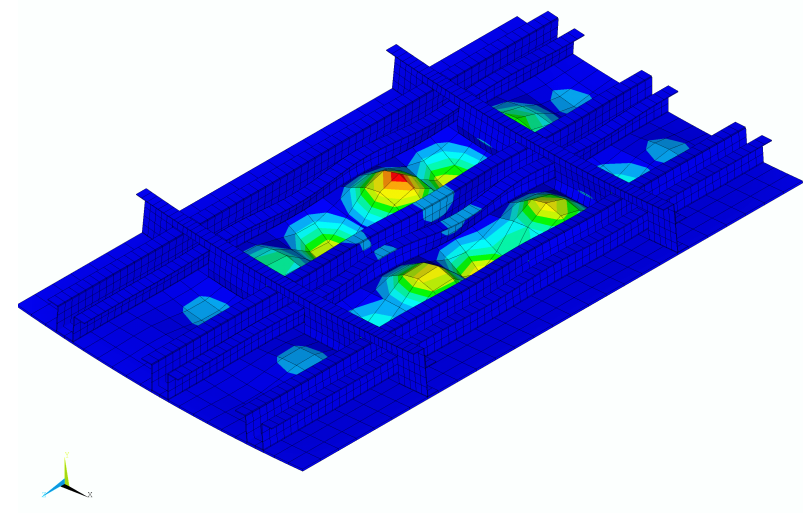

(c) Bottom sector panel

Figure 13: Normalised displacement field of the first buckling mode of the local models for the optimum solution.

The obtained results for the presented design case seem realistic and coherent: at the component scale, the elements composing the stiffened panel (of the most critical ZOIs of the fuselage barrel) buckle simultaneously in agreement with well-established design procedures used in both academic and industrial communities [46]. Moreover, difference between the mass of the optimised configuration provided by the MSO and that of the reference solution (taken from the literature and obtained by using simplifying hypotheses and simple models and rules) is lower than $1.2 \%$. However, the reference solution taken from the literature is infeasible and does not meet some of the design criteria employed in this work which result too restrictive. This constitutes a sort of "numerical proof" about the unsuitability of some simplifying hypotheses and low-fidelity analytical/numerical models often used in the preliminary design phase of aircraft structures.

These results encourage research activity in this direction. As far as perspectives of this work are concerned, the formulation of the CNLPP will be enhanced by adding requirements on the postbuckling behaviour of the most critical stiffened panel in each sector of the fuselage barrel. Moreover, 
research is ongoing in order to extend the MSO approach to the case of thin-walled structures made of composite materials. Of course, in this case the design problem formulation must integrate requirements and specificities of composite solutions, e.g. suitable failure criteria at each scale (lamina-level and constitutive phases-level), delamination criteria, manufacturing requirements, etc.

\section{Appendix A. Mesh sensitivity analysis}

The sensitivity analysis to the mesh parameters has been conducted by looking at the various mechanical responses of both the GFEM and the LFEM when LSol is evaluated. The mesh parameters considered are the element class, i.e. linear (L) or quadratic (Q), and the mesh size for both GFEM and LFEM. Because of the way the GL modelling approach is implemented, both global and local mesh parameters affect the local responses, while global ones only depend upon global mesh parameters. All the various combinations of the parameters have been evaluated, but, for the sake of brevity, only the most important results are reported here.

Concerning the GFEM, the mesh has been parametrised in terms of a single sizing parameter, i.e. the number of elements between two adjacent stringers (indicated with a number following the letter $\mathrm{L} / \mathrm{Q}$ in the results). An automatic check has been implemented to prevent the generation of highly distorted shell elements with a threshold aspect ratio equal to two. The effect of the GFEM mesh parameters on one of the global scale responses (the rotation $\theta_{\mathrm{X}}$ of section $\mathrm{B}$ under LC1) and on a local scale one (the value $\lambda^{\text {Top }}$ of the buckling factor of most critical stiffened panel of the top sector under LC8) is reported in Tab. A.1. To obtain these results, the mesh of the LFEM is fixed to the mesh parameters set LFEM-Q1 reported in Tab. A.2 (more details on the sensitivity analysis of the LFEM responses are given in the following of this section). Derived informations are reported in Figs. A.1 and A.2. For these analyses, the total execution time has been normalised with respect to the smallest one (obtained using one linear element between stringers, i.e. mesh parameters set GFEM-L1), while the mechanical responses have been normalised with respect to fully converged ones (obtained with mesh parameters set GFEM-Q4). As it can be seen,

\begin{tabular}{lrrrr}
\hline Mesh par. & DOFs $^{\dagger}$ & Exec. time $^{*}[\mathrm{~s}]$ & $\theta_{\mathrm{x}}[\mathrm{rad}]$ & $\lambda^{\text {Top }}[-]$ \\
\hline GFEM-L1 & 10476 & 36 & $1.1301 \cdot 10^{-3}$ & 0.5675 \\
GFEM-L2 & 28428 & 40 & $1.1305 \cdot 10^{-3}$ & 0.5658 \\
GFEM-L3 & 53760 & 44 & $1.1326 \cdot 10^{-3}$ & 0.5640 \\
GFEM-L4 & 100752 & 51 & $1.1333 \cdot 10^{-3}$ & 0.5632 \\
GFEM-L5 & 144498 & 60 & $1.1339 \cdot 10^{-3}$ & 0.5628 \\
GFEM-Q1 & 28140 & 38 & $1.1307 \cdot 10^{-3}$ & 0.5651 \\
GFEM-Q2 & 78336 & 42 & $1.1342 \cdot 10^{-3}$ & 0.5630 \\
GFEM-Q3 & 150546 & 46 & $1.1338 \cdot 10^{-3}$ & 0.5629 \\
GFEM-Q4 & 287988 & 63 & $1.1341 \cdot 10^{-3}$ & 0.5627 \\
\hline f Active degrees of freedom of the GFEM. \\
${ }^{*}$ Total execution time including LFEM analyses. \\
\multicolumn{5}{l}{}
\end{tabular}

Table A.1: Mesh sensitivity of the GFEM, main results. Local mesh set at LFEM-Q1.

the improvement obtainable by using more accurate meshes than GFEM-L1 in the evaluation of mechanical responses is rather small.

In this case, the choice of the mesh parameters set GFEM-Q1 is motivated by its ability to perfectly model the curved shape of the fuselage barrel components, with a small increase in computational time.

Also in the case of LFEM, the mesh has been parametrised in terms of a single sizing parameter. Each of the edges of the geometry describing the components has been divided in a given amount 


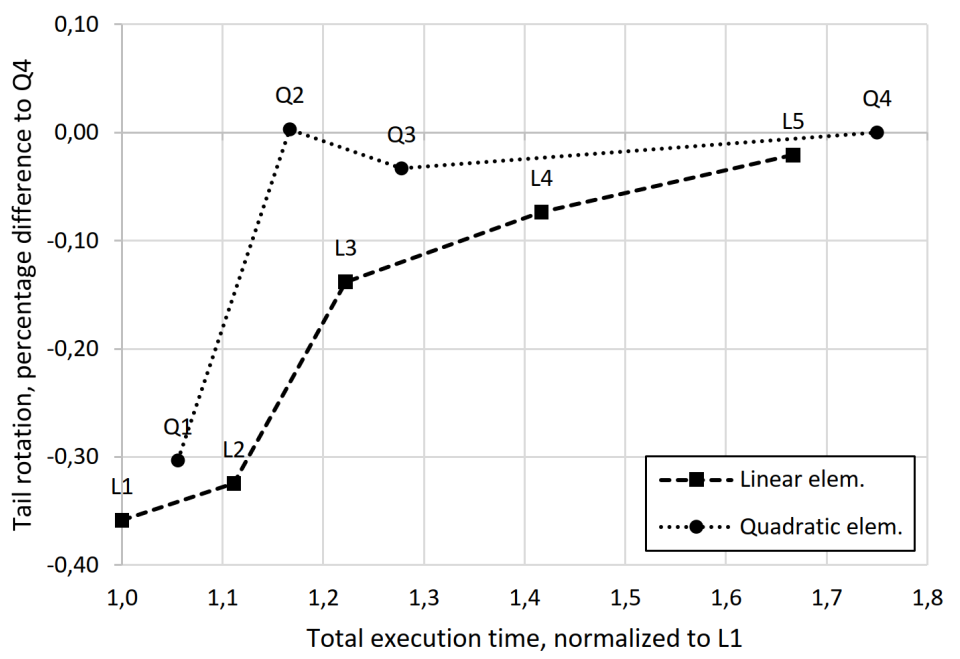

Figure A.1: Sensitivity to the GFEM mesh parameters of the percentage difference of the rotation of section B with respect to the one obtained with mesh GFEM-Q4.

of mesh units. The assumption is that the buckling could occur in any one of the plate-regions composing the structure. Therefore, the numbers of mesh units must be chosen to ensure a homogeneous level of accuracy in the estimation of the buckling load in accordance with the first expected buckling mode of each plate. For example, when looking at the stringers cross section, the free flanges have been divided in one mesh unit, the webs in three mesh unit and the bonded flanges in two mesh unit. Finally, automatic checks have been implemented to ensure that the final elements have nearly unitary aspect ratios. With this parametrisation set, the number of element in each mesh unit and their element class (linear, L, or quadratic, Q) constitute the mesh parameters of the sensibility analysis. The results of such analysis are reported in Tab. A.2 and Figs. A.3, A.4 and A.5. To obtain these results, the mesh of the GFEM is fixed to the mesh parameters set GFEM-Q1. In this case, the use of a light mesh composed of few linear shape functions elements,

\begin{tabular}{lrrrrr}
\hline Mesh par. & DOFs $^{\dagger}$ & Exec. time $^{*}[\mathrm{~s}]$ & $\lambda^{\text {Top }}[-]$ & $\lambda^{\text {Lat }}[-]$ & $\lambda^{\text {Bot }}[-]$ \\
\hline LFEM-L1 & 39396 & 23 & 0.6243 & 0.3435 & 0.6122 \\
LFEM-L2 & 131268 & 40 & 0.5783 & 0.2976 & 0.5502 \\
LFEM-L3 & 294048 & 75 & 0.5691 & 0.2864 & 0.5327 \\
LFEM-L4 & 498192 & 133 & 0.5646 & 0.2822 & 0.5278 \\
LFEM-L5 & 784056 & 222 & 0.5626 & 0.2801 & 0.5247 \\
LFEM-Q1 & 113880 & 38 & 0.5651 & 0.2886 & 0.5297 \\
LFEM-Q2 & 385608 & 104 & 0.5555 & 0.2764 & 0.5186 \\
LFEM-Q3 & 870000 & 261 & 0.5556 & 0.2760 & 0.5188 \\
\hline Active degrees of freedom of the LFEM. \\
* Total execution time including GFEM analysis.
\end{tabular}

Table A.2: Mesh sensitivity of the LFEM. Global mesh set at GFEM-Q1.

like LFEM-L1, shows all its limitations. For LFEM-L1 the percentage difference in the computed buckling factor reaches $25 \%$ with respect to the results obtained by a fully converged mesh like LFEM-Q3.

By considering a threshold percentage difference of $5 \%$, the best compromise between accuracy and computational time is obtained by adopting the mesh parameters set LFEM-Q1. 


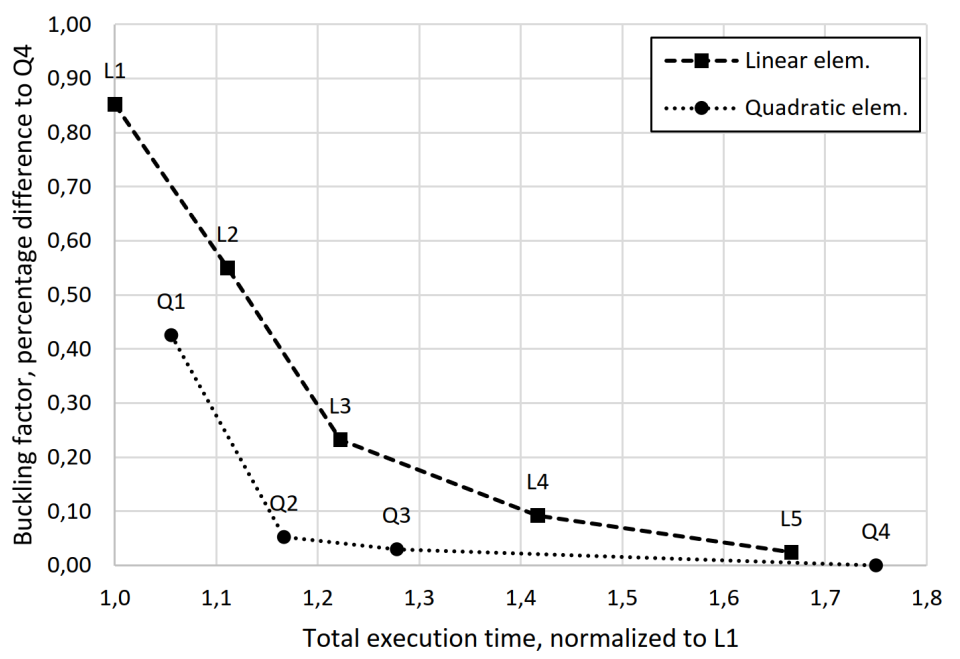

Figure A.2: Sensitivity to the GFEM mesh parameters of the percentage difference of the buckling factor the most critical stiffened panel in the top sector with respect to the one obtained with mesh GFEM-Q4.

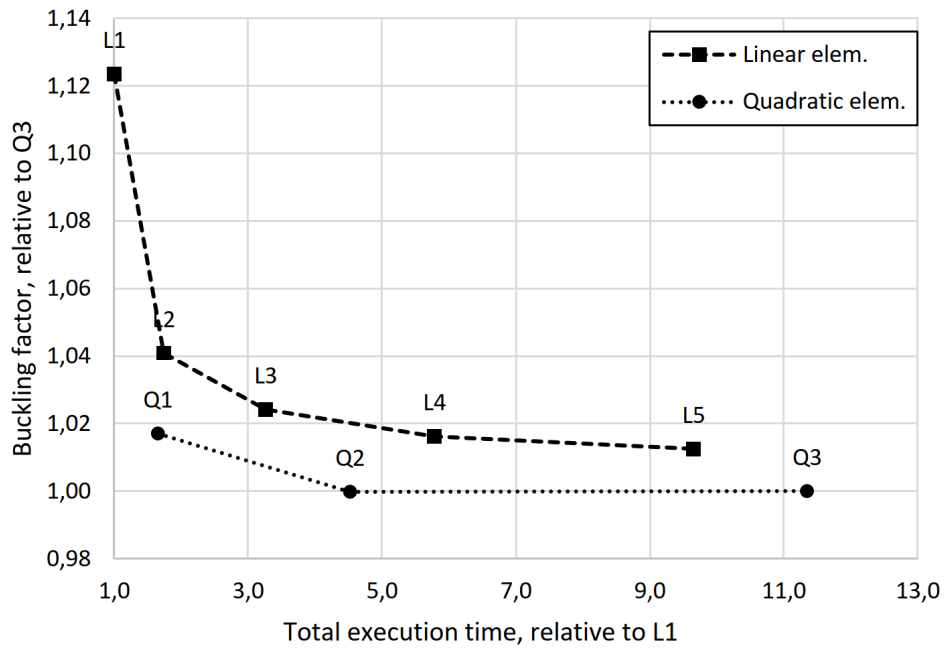

Figure A.3: Sensitivity to the LFEM mesh parameters of the buckling factor of the most critical stiffened panel of the top sector (normalised with respect to that obtained with mesh LFEM-Q4). 


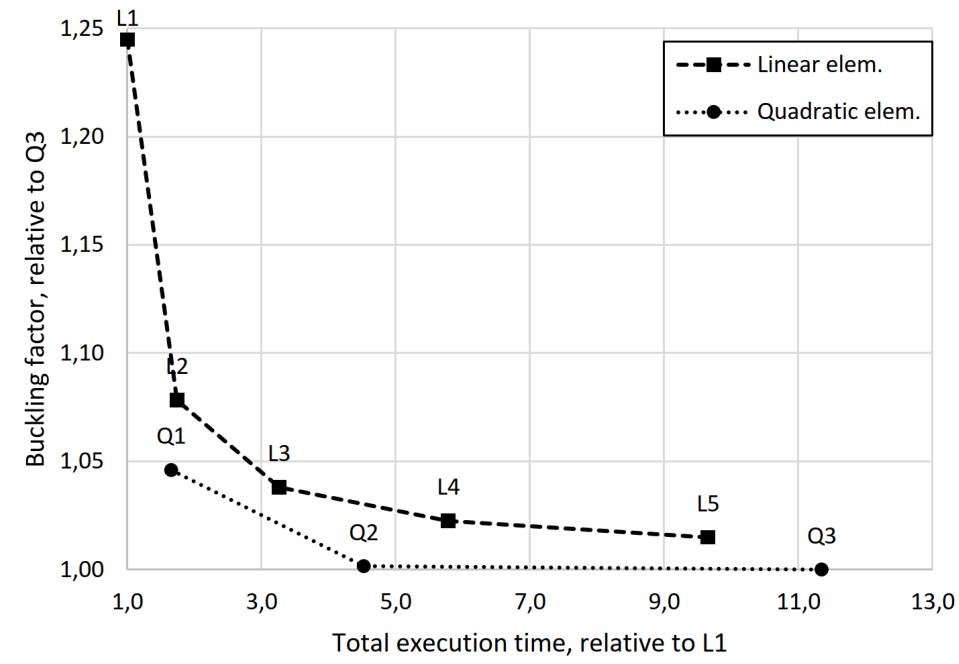

Figure A.4: Sensitivity to the LFEM mesh parameters of the buckling factor of the most critical stiffened panel of the lateral sector (normalised with respect to that obtained with mesh LFEM-Q4).

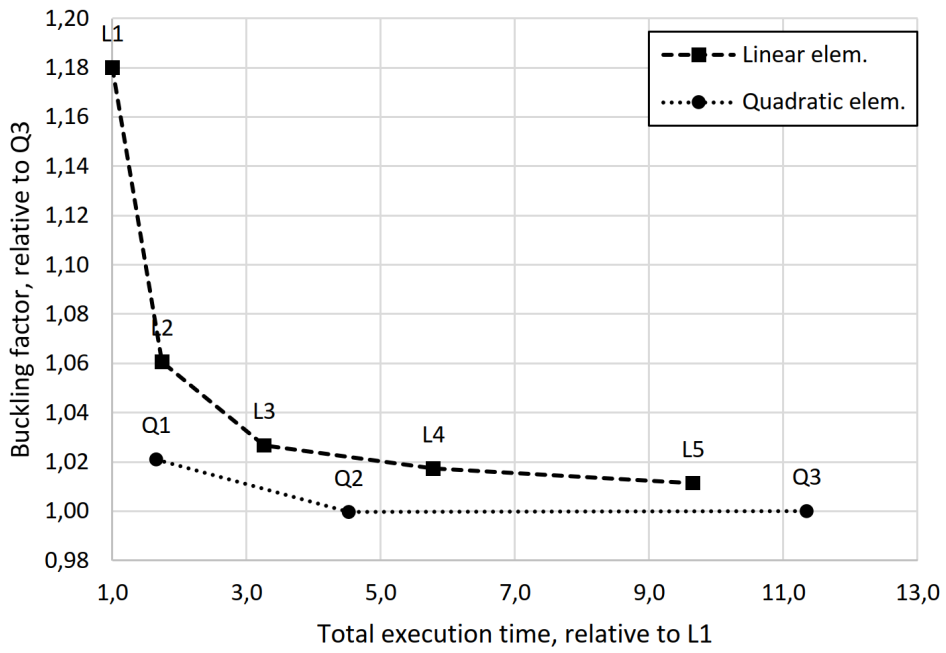

Figure A.5: Sensitivity to the LFEM mesh parameters of the buckling factor of the most critical stiffened panel of the bottom sector (normalised with respect to that obtained with mesh LFEM-Q4). 


\section{References}

[1] J.E. Wignot, H. Combs, and A.F. Ensrud. Analysis of circular shell-supported frames. Technical note 929, NACA, 1944.

[2] P. Kuhn, J.P. Peterson, and L.R. Levin. Summary of diagonal tension. Technical note 2661, NACA, 1952.

[3] G. Gerard. The Crippling Strength of Compression Elements. Journal of the Aerospace Sciences, 25(1):37-52, 1958.

[4] D. Williams. An introduction to the theory of aircraft structures. Arnold, 1960.

[5] R.M. Rivello. Theory and analysis of flight structures. McGraw-Hill College, 1969.

[6] M.C.Y. Niu. Airframe structural design: practical design information and data on aircraft structures. Conmilit Pr, 1988.

[7] E.F. Bruhn. Analysis and design of flight vehicle structures. Tri-State Offset Co., 1973.

[8] B.K. Anderson and T.T. Takahashi. Conceptual fuselage design with direct CAD modeling. In 17th AIAA Aviation Technology, Integration, and Operations Conference, Denver, Colorado, 2017.

[9] S. Grihon, M. Samuelides, A. Merval, A. Remouchamps, M. Bruyneel, B. Colson, and K. Hertel. Fuselage structure optimisation. In Advances in collaborative civil aeronautical multidisciplinary design optimization, Progress in astronautics and aeronautics. AIAA, 2009.

[10] L. Boni and D. Fanteria. Development of analytical methods for fuselage design: validation by means of finite element analyses. Proceedings of the Institution of Mechanical Engineers, Part G: Journal of Aerospace Engineering, 218(5):315-327, 2004.

[11] L. Boni. Methodologies for the optimum design of fuselage structures of transport aircraft. $\mathrm{PhD}$ thesis, University of Pisa, Pise, Italy, 2004.

[12] J. Loughlan. The buckling performance of composite stiffened panel structures subjected to combined in-plane compression and shear loading. Composite Structures, 29(2):197 - 212, 1994.

[13] O.F. Hughes, B. Ghosh, and Y. Chen. Improved prediction of simultaneous local and overall buckling of stiffened panels. Thin-Walled Structures, 42(6):827 - 856, 2004.

[14] D.G. Stamatelos, G.N. Labeas, and K.I. Tserpes. Analytical calculation of local buckling and post-buckling behavior of isotropic and orthotropic stiffened panels. Thin-Walled Structures, 49(3):422 - 430, 2011.

[15] J. Sobieszczanski and D. Loendorf. A Mixed Optimization Method for Automated Design of Fuselage Structures. Journal of Aircraft, 9(12):805-811, December 1972.

[16] M. Fischer, D. Kennedy, and C.A. Featherston. Multilevel framework for optimization of lightweight structures. Proceedings of the Institution of Mechanical Engineers, Part G: Journal of Aerospace Engineering, 226(4):380-394, April 2012. 
[17] W.J. Vankan, B.A.T. Noordman, and K. Kueres. High and low fidelity finite element modelling in aircraft composite fuselage structural analysis and optimisation. Technical report 2013-197, National Aerospace Laboratory NLR, 2014.

[18] S. Venkataraman and R.T. Haftka. Structural optimization complexity: what has moore's law done for us? Structural and Multidisciplinary Optimization, 28(6):375-387, 2004.

[19] I. Hirai, B.P. Wang, and W.D. Pilkey. An efficient zooming method for finite element analysis. International Journal for Numerical Methods in Engineering, 20(9):1671-1683, 1984.

[20] I. Hirai, Y. Uchiyama, Y. Mizuta, and W.D. Pilkey. An exact zooming method. Finite Elements in Analysis and Design, 1(1):61-69, 1985.

[21] C.C. Jara-Almonte and C.E. Knight. The specified boundary stiffness/force SBSF method for finite element subregion analysis. International Journal for Numerical Methods in Engineering, 26(7):1567-1578, 1988.

[22] C.T. Sun and K.M. Mao. A global-local finite element method suitable for parallel computations. Computers \&5 Structures, 29(2):309-315, 1988.

[23] K.M. Mao and C.T. Sun. A refined global-local finite element analysis method. International Journal for Numerical Methods in Engineering, 32(1):29-43, 1991.

[24] J.D. Whitcomb. Iterative global/local finite element analysis. Computers \& Structures, 40(4):1027-1031, 1991.

[25] N.G. Cormier, B.S. Smallwood, G.B. Sinclair, and G. Meda. Aggressive submodelling of stress concentrations. International Journal for Numerical Methods in Engineering, 46(6):889-909, 1999 .

[26] L. Gendre, O. Allix, P. Gosselet, and F. Comte. Non-intrusive and exact global/local techniques for structural problems with local plasticity. Computational Mechanics, 44(2):233-245, 2009.

[27] P.A. Guidault, O. Allix, L. Champaney, and J.P. Navarro. A two-scale approach with homogenization for the computation of cracked structures. Computers $\& 5$ Structures, 85(17-18):13601371, 2007.

[28] M. Akterskaia, E. Jansen, S. Hühne, and R. Rolfes. Efficient progressive failure analysis of multi-stringer stiffened composite panels through a two-way loose coupling global-local approach. Composite Structures, 183:137-145, 2018.

[29] M. Montemurro. A contribution to the development of design strategies for the optimisation of lightweight structures. HDR thesis. Université de Bordeaux, Bordeaux, France, 2018.

[30] L. Boni and D. Fanteria. Finite-element-based assessment of analytical methods for the design of fuselage frames. Proceedings of the Institution of Mechanical Engineers, Part G: Journal of Aerospace Engineering, 220(5):387-398, 2006.

[31] Metallic materials and elements for aerospace vehicle structures MIL-HDBK-5j. Technical report, Departement of defence - United States of America, 2003.

[32] Certification specifications and acceptable means of compliance for large aeroplanes CS-25. Technical report, Official Publication of the European Aviation Safety Agency, 2018. 
[33] L. Dai, H. Chen, Y. Zhang, X. Wang, X. Feng, Y. Bian, and G. Yang. An extended method of estimating the fatigue performance of mechanical structures with fasteners subject to shear loads. Advances in Mechanical Engineering, 10(4):1-11, 2018.

[34] M. Montemurro and A. Catapano. A general b-spline surfaces theoretical framework for optimisation of variable angle-tow laminates. Composite Structures, 209:561 - 578, 2019.

[35] M. Montemurro, M.I. Izzi, J. El-Yagoubi, and D. Fanteria. Least-weight composite plates with unconventional stacking sequences: Design, analysis and experiments. Journal of Composite Materials, 2019 in press.

[36] E. Panettieri, M. Montemurro, and A. Catapano. Blending constraints for composite laminates in polar parameters space. Composites Part B: Engineering, 168:448 - 457, 2019.

[37] G. Costa, M. Montemurro, and J. Pailhès. A General Hybrid Optimization Strategy for Curve Fitting in the Non-Uniform Rational Basis Spline Framework. Journal of Optimization Theory and Applications, 176(1):225-251, 2018.

[38] G. Bertolino, M. Montemurro, and G. De Pasquale. Multi-scale shape optimisation of lattice structures : an evolutionary-based approach. International Journal on Interactive Design and Manufacturing, 13(4):1565-1578, 2019.

[39] Lorenzo Cappelli, Marco Montemurro, Frédéric Dau, and Laurent Guillaumat. Characterisation of composite elastic properties by means of a multi-scale two-level inverse approach. Composite Structures, 204:767 - 777, 2018.

[40] Y. Audoux, M. Montemurro, and J. Pailhes. A surrogate model based on non-uniform rational b-splines hypersurfaces. Procedia CIRP, 70:463 - 468, 2018. 28th CIRP Design Conference 2018, 23-25 May 2018, Nantes, France.

[41] M. Montemurro and A. Catapano. On the effective integration of manufacturability constraints within the multi-scale methodology for designing variable angle-tow laminates. Composite Structures, 161:145 - 159, 2017.

[42] Ansys. ANSYS Mechanical APDL: Contact Technology Guide. Release 18.1. ANSYS, Inc., Southpointe, 2600 ANSYS Drive, Canonsburg, PA 15317, 2017.

[43] S. Timoshenko and J. Gere. Theory of elastic stability. McGraw-Hill Book Company inc., New York and London, 2nd edition, 1961.

[44] S.B. Batdorf and M. Stein. Critical combinations of shear and direct stress for simply supported rectangular flat plates. Technical Note 1223, NACA, 1947.

[45] M. Montemurro, A. Vincenti, and P. Vannucci. The automatic dynamic penalisation method (ADP) for handling constraints with genetic algorithms. Computer Methods in Applied Mechanics and Engineering, 256:70-87, 2013.

[46] G. Gerard. Minimum weight analysis of compression structures. New York University Press, 1956. 